\title{
Uncertainty analysis of force coefficients during micromilling of titanium alloy
}

\author{
Erman Gözï ${ }^{1} \cdot$ Yiğit Karpat $^{1,2,3}$
}

Received: 20 February 2017 / Accepted: 15 May 2017 /Published online: 30 May 2017

(C) Springer-Verlag London 2017

\begin{abstract}
Predicting process forces in micromilling is difficult due to complex interaction between the cutting edge and the work material, size effect, and process dynamics. This study describes the application of Bayesian inference to identify force coefficients in the micromilling process. The Metropolis-Hastings (MH) algorithm Markov chain Monte Carlo (MCMC) approach has been used to identify probability distributions of cutting, edge, and ploughing force coefficients based on experimental measurements and a mechanistic model of micromilling. The Bayesian inference scheme allows for predicting the upper and lower limits of micromilling forces, providing useful information about stability boundary calculations and robust process optimization. In the first part of the paper, micromilling experiments are performed to investigate the influence of micromilling process parameters on machining forces, tool edge condition, and surface texture. Under the experimental conditions used in this study, built-up edge formation is observed to have a significant influence on the process outputs in micromilling of titanium alloy Ti6A14V. In the second part, Bayesian inference was explained in detail and applied to model micromilling force prediction. The force predictions are validated with the experimental measurements. The paper concludes with a discussion of the effectiveness of
\end{abstract}

Yiğit Karpat

ykarpat@bilkent.edu.tr

1 Department of Industrial Engineering, Bilkent University, Bilkent, Ankara, Turkey

2 Micro System Design and Manufacturing Center, Department of Mechanical Engineering, Bilkent University, Bilkent,

Ankara, Turkey

3 UNAM-Institute of Materials Science and Nanotechnology, Ankara, Turkey employing Bayesian inference in micromilling force modeling considering special machining cases.

Keywords Micromilling $\cdot$ Mechanistic modeling $\cdot$ Bayesian inference $\cdot$ Markov chain Monte Carlo $\cdot$ Uncertainty analysis

\section{Nomenclature}

$\alpha_{\mathrm{e}} \quad$ Rake angle

$A_{\mathrm{p}} \quad$ Ploughed area

$d F_{\mathrm{t}_{j}} \quad$ Differential tangential cutting force corresponding to cutting edge $j$

$d F_{\mathrm{r}_{j}} \quad$ Differential radial cutting force corresponding to cutting edge $j$

$d F_{\mathrm{tp}_{j}} \quad$ Differential tangential ploughing force

$d F_{\mathrm{rp}_{j}} \quad$ Differential radial ploughing force

$d F_{\mathrm{x}_{j}} \quad$ Measured micromilling forces in the $\mathrm{X}$ direction corresponding to cutting edge $j$

$d F_{\mathrm{y}_{j}} \quad$ Measured micromilling forces in the $\mathrm{Y}$ direction corresponding to cutting edge $j$

$d z \quad$ Differential height element

$\varphi \quad$ Cutting angle

$F_{\text {measured }}$ Instantaneous experimental cutting force data point

$F_{\text {predicted }}$ Predicted cutting force data point

$h \quad$ Uncut chip thickness in milling

$h_{\mathrm{c}} \quad$ Critical chip thickness

$h_{j} \quad$ Uncut chip thickness corresponding to cutting edge $j$

$K_{\text {te }} \quad$ Tangential edge coefficient

$K_{\text {tc }} \quad$ Tangential cutting coefficient

$K_{\text {re }} \quad$ Radial edge coefficient

$K_{\text {rc }} \quad$ Radial cutting coefficient

$K_{\text {tp }} \quad$ Tangential ploughing coefficient

$K_{\mathrm{rp}} \quad$ Radial ploughing coefficient 
$r \quad$ Edge radius

Sa Areal surface roughness

Ssk Surface skewness

Sku Surface kurtosis

$\mathrm{Sq} \quad$ Root-mean-square height

$t_{\mathrm{u}} \quad$ Uncut chip thickness in 2D cutting

$V_{\mathrm{p}} \quad$ Ploughing volume

$\bar{F}_{x, m} \quad$ Measured average forces in the $x$ direction

$\bar{F}_{y, m} \quad$ Measured average forces in the $y$ direction

\section{Introduction}

Micromechanical milling is an effective technique to produce microcomponents having three-dimensional surfaces made from engineering materials $[1,2]$. Controlling the dimensional tolerances and surface quality of microcomponents considering productivity issues is a challenge. Predictive process models, which have been successfully applied to macroscale milling, would be helpful to assist in selecting stable machining conditions, estimating surface location errors, and optimizing process parameters in micromilling [3, 4]. Extending such predictive models to micromilling is not a straightforward task due to work material size effects, tool run-out, tool wear, built-up edge, and difficulties in identifying structural dynamic parameters. It is difficult to deal with the stochastic behavior of the micromilling process and uncertainties introduced by these factors using deterministic predictive process models. This paper describes the application of Bayesian inference methods to micromilling force modeling.

Force modeling based on process input parameters is usually considered as the first step in process modeling. Mechanistic process modeling is usually preferred to model process forces in micromilling where the relationship between the work material and the cutting edge is obtained through cutting, edge, and ploughing force coefficients [5-7]. These coefficients are often calculated from either experimental measurements of process forces or finite element-based simulation models [8]. The elasto-plastic behavior of the work material, tool cutting edge radius, tool deflections, tool runout, and tool vibrations all affect the machining forces, leading to uncertainties in micromilling forces especially when machining a difficult-to-cut material such as titanium alloy. Titanium alloy Ti6Al4V is a popular material in the biomedical industry due its bio-compatibility and low density.

There are uncertainties associated with cutting, edge, and ploughing force coefficients. When micromilling forces are calculated based on these coefficients, providing uncertainty information would be useful for the users. In a recent study, Karandikar et al. [9] demonstrated application of Bayesian inference to milling force modeling. They used the Markov chain Monte Carlo (MCMC) method to calculate the posterior distributions of the force coefficients. They concluded that
Bayesian inference improves the predictive capability compared to linear regression-based traditional methods. Karandikar et al. [10,11] also used Bayesian inference to model tool life in milling and turning operations. Niaki et al. [12] demonstrated the use of Bayesian inference in a mechanistic model of the tool wear while machining nickel-based alloys. Mehta et al. [13] used the Bayesian parameter inference method to model machining forces. Cao and $\mathrm{Li}$ [14] emphasized the importance of uncertainties in selecting stable machining conditions in micromilling. Jaffery et al. [15] studied the influence of micromachining process parameters by considering the relationship between undeformed chip thickness and tool edge radius. They found that when the feed is set above the tool edge radius, the feed rate is the most important parameter affecting tool wear, surface roughness, and burr width. When the undeformed chip thickness is set less than the tool edge radius, the influence of the feed on tool wear, surface roughness, and burr width was observed to be lower. Jaffery et al. [16] also pointed out that tool wear is driven by stochastic factors.

In this study, the MCMC method [17-19] has been applied to the micromilling process to describe uncertainties in force predictions. A mechanistic micromilling model from the literature [5] has been adopted, and the probability distributions of cutting, edge, and ploughing force coefficients were calculated. As a result of uncertainty analysis, the mean and standard deviations of the micromilling forces can be estimated.

Built-up edge (BUE) formation is a common issue during micromachining of ductile materials affecting the process outputs. The size and stability of BUE depends on the machining conditions. In some cases, it is known to protect the cutting edge from rapid wear, but it has a detrimental effect on the surface finish, which is quite important in micromachining [20]. In this study, BUE is investigated in detail and considered as an important source of uncertainty.

The paper is organized as follows. First, observations of process outputs such as micromilling forces, tool edge condition, and surface texture are investigated. Second, mechanistic micromilling process modeling technique is explained. Third, Bayesian inference is applied to calculate probability distributions of force coefficients. Fourth, the results are discussed.

\section{Experimental details}

The micromachining experiments were performed on a CNC milling center DMG HSC 55 together with a NSK HES 510 high-speed spindle ( $\max 50,000 \mathrm{rpm})$ as shown in Fig. 1a. The work material is selected as titanium alloy Ti6AL4V due to its popularity in practice and research. The workpiece is $80 \mathrm{~mm}$ long and its width is $37 \mathrm{~mm}$. The titanium work material has a lamellar structure consisting of $80 \%$ alpha and $20 \%$ beta phases as shown in Fig. 1b. Slot micromilling experiments 
Fig. 1 a Experimental setup of milling experiments. $\mathbf{b}$

Microstructure of the material

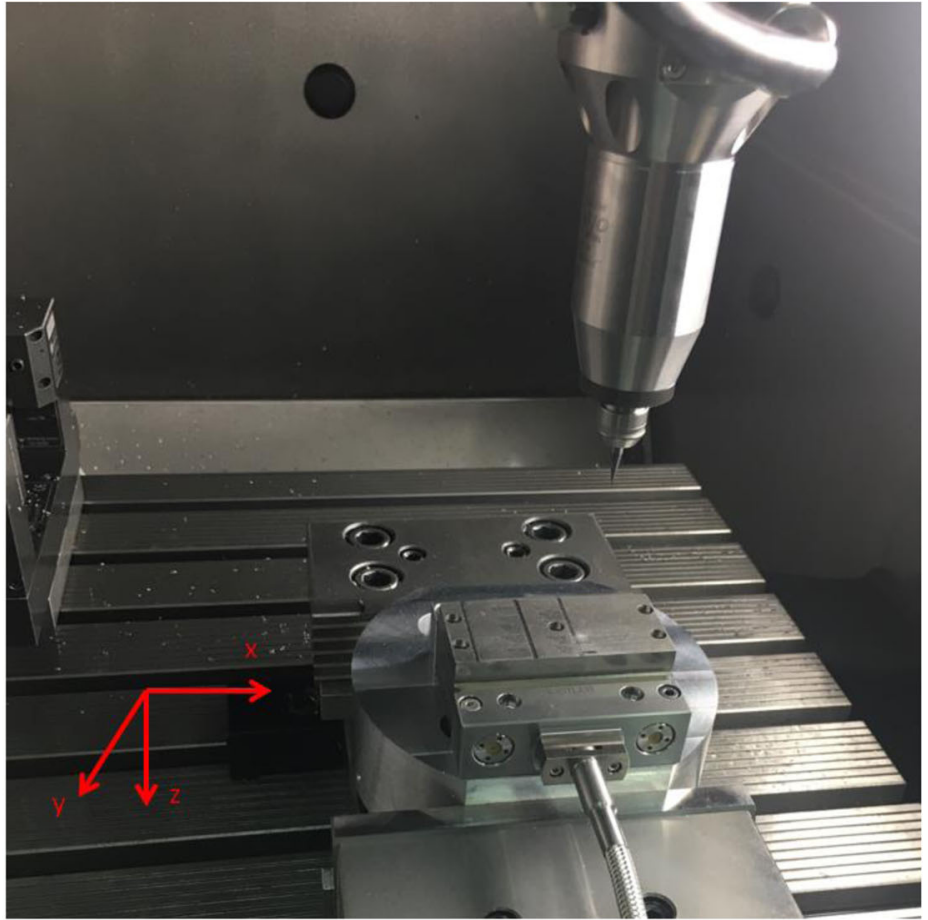

(a)

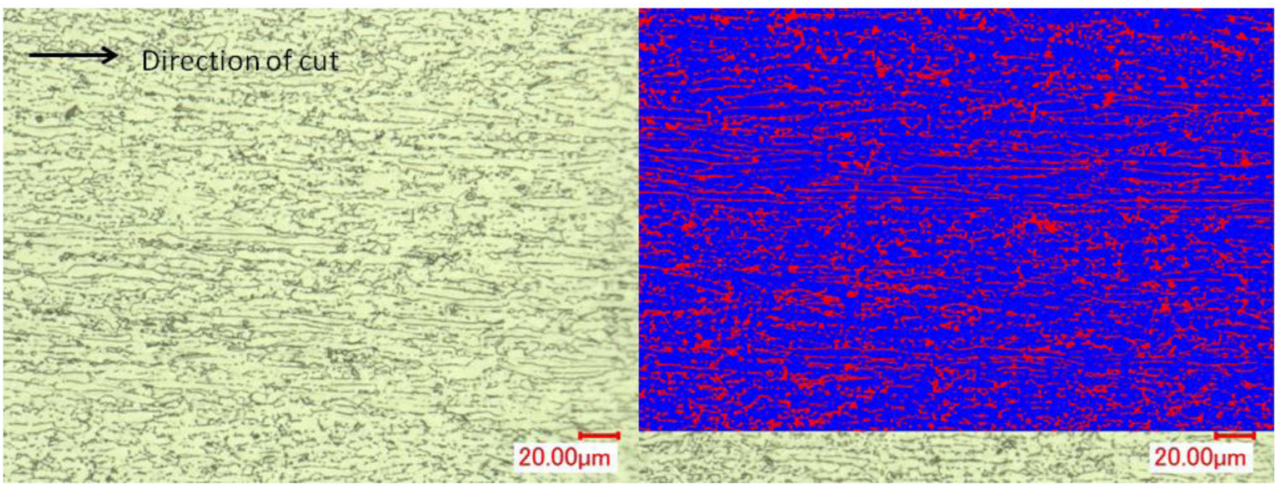

(b) were performed under dry conditions. Machining forces were measured using a Kistler mini dynamometer (9256 C1) with its charge amplifier (5080A). The measurements were transferred to a PC using a data acquisition system (National Instruments). Force signals were acquired with $10^{5}$ data points per second during the experiments. Micro end mills (NS Tools MSE $2300.4 \times 0.8$ ) having a $0.4-\mathrm{mm}$ diameter and $2-\mu \mathrm{m}$ cutting edge radius were used in the experiments. The cutting speed was kept constant at $35 \mathrm{~m} / \mathrm{min}$, which corresponds to 28,000-rpm spindle speed. As the micro end mill diameter decreases, the rotational speed of the microtool must be increased in order to attain an acceptable cutting speed. However, the tooth passing frequency should not exceed the bandwidth of the dynamometer [20]. A special attention was paid to the placement of the micro end mill to the tool holder; the static run-out was measured to be less than $1 \mu \mathrm{m}$ using the
Mahr Millimar C-series indicator. The duration of cut (10 s) was kept the same in all experiments. Micromachining conditions are given in Table 1. The axial depth of cut was set as $10 \%$ of the nominal tool diameter. Feed per tooth values were set considering the edge radius of the end mill. Force measurements during the micromilling experiments, the condition of the tool edge after the experiments, and the resulting surface texture have been investigated in detail.

\section{Experimental observations of process outputs}

\subsection{Micromilling force measurements}

Figure $2 \mathrm{a}$ shows the micromilling forces in $\mathrm{x}, \mathrm{y}$, and $\mathrm{z}$ directions for a feed value of $0.4 \mu \mathrm{m} /$ tooth. The variation peak 
Table 1 Machining conditions for micromilling of Ti6AL4V

\begin{tabular}{llll}
$\begin{array}{l}\text { Rotational } \\
\text { speed }(\mathrm{rpm})\end{array}$ & $\begin{array}{l}\text { Cutting speed } \\
(\mathrm{m} / \mathrm{min})\end{array}$ & $\begin{array}{l}\text { Axial depth of } \\
\text { cut }(\mu \mathrm{m})\end{array}$ & $\begin{array}{l}\text { Feed per } \\
\text { tooth }(\mu \mathrm{m} / \mathrm{rev})\end{array}$ \\
\hline 28,000 & 35 & 40 & $\begin{array}{c}0.4,0.6,0.8,1,1.2, \\
1.5,2,3,4\end{array}$
\end{tabular}

forces and different directions can also be identified (as shown with "o") in this figure. Peak forces are important in micromilling since calculating the mean forces does not yield satisfactory information about the process conditions. The peak forces were counted using the data acquisition software NI DIAdem for the whole duration of the process. Peak-tovalley forces in positive and negative directions were calculated for all feed values, and the mean values are shown in Fig. 2b. Peak-to-valley forces decrease down to $1 \mu \mathrm{m} /$ tooth feed, and around this feed value, "force instability" occurs where the peak-to-valley forces slightly increase and start decreasing again around $0.6 \mu \mathrm{m} /$ tooth. Based on these results, feed above $1 \mu \mathrm{m} /$ tooth can be identified as the shearingdominated zone, feed between 0.6 and $1 \mu \mathrm{m} /$ tooth can be identified as the transition zone, and feed less than $0.6 \mu \mathrm{m} /$ tooth can be identified as the ploughing-dominated zone.

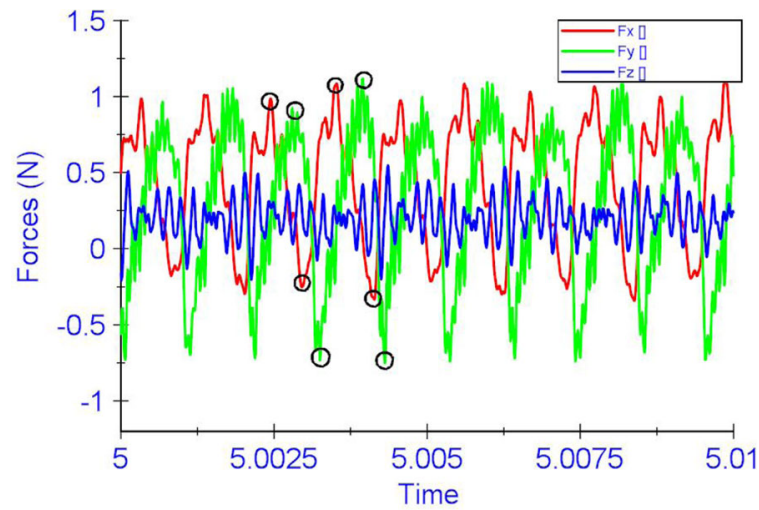

(a)

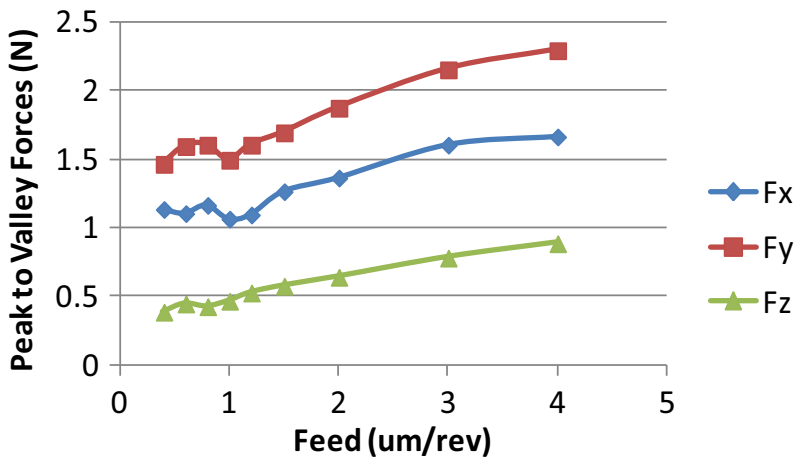

(b)

Fig. 2 a Acquired force signals from the micromilling experiments, $0.4 \mu \mathrm{m} /$ tooth. b Peak-to-valley forces for different feed values

\subsection{Tool edge condition}

Figure $3 \mathrm{a}$ shows the edge condition at the end of slot micromilling tests, which reveals that a built-up edge (BUE) at both cutting edges was formed during machining tests. The same micro end mill was used in all machining experiments in order to keep experimental conditions constant (tool overhang length, run-out, etc.). BUE is known to be detrimental to surface quality, but if it is stable and small in size, it may help to protect the cutting edge. Figure $3 \mathrm{~b}$ shows the image of the cutting edge scanned with a confocal laser microscope (Keyence VHX-110). The cutting edge has a chamfered tip, which increases the strength of the tip and also promotes work material accumulation in front of the tool which acts like the cutting edge. BUE changes the rake and clearance angles of the cutting edge, and its size depends on the machining conditions [21]. It is possible to eliminate BUE by increasing the cutting speed, but in micromilling, it is limited by the maximum spindle speed and dynamics of the process. A high cutting speed would also result in faster tool wear, especially when difficult-to-cut materials are machined. Figure $3 \mathrm{c}$ shows the edges of the micro end mill after BUE is removed by applying a cleaning procedure. The edge radii were measured to increase from initial 2 to $4 \mu \mathrm{m}$. A rapid increase in edge radius during the break-in period increases the possibility of material entrapment in front of the cutting edge.

In Fig. 4, two possible cases of micromachining configurations are shown. If there is no BUE in front of the cutting edge and assuming that the uncut chip thickness $t_{\mathrm{u}}$ is smaller than the edge radius $r$, then the effective rake becomes negative $\alpha_{\mathrm{e}}$. The material underneath the cutting edge is ploughed during machining. This volume is a function of material elastic properties. It is difficult to model material behavior under such conditions, which introduce uncertainty to modeling. If there is BUE formation in front of the tool, the ploughing forces are applied to it. The resulting rake angle becomes positive, and the contact conditions between the cut material and the tool material cease. The condition of the edge directly influences the generated surface. The stability of BUE and its size and shape introduce uncertainty to modeling. Predicting BUE stability, size, and shape depending on the machining conditions is a challenging task [21].

\subsection{Surface texture investigation}

The condition of the cutting edge and feed value directly influences machined surface properties. Therefore, investigation of the surface texture would yield some useful information about the combined effect of BUE and feed on the surface texture. In this study, micromilled surfaces were investigated by considering areal surface properties. Three-dimensional topography of the micromilled surfaces was obtained by using a laser scanning microscope (Keyence VK-X110). The 

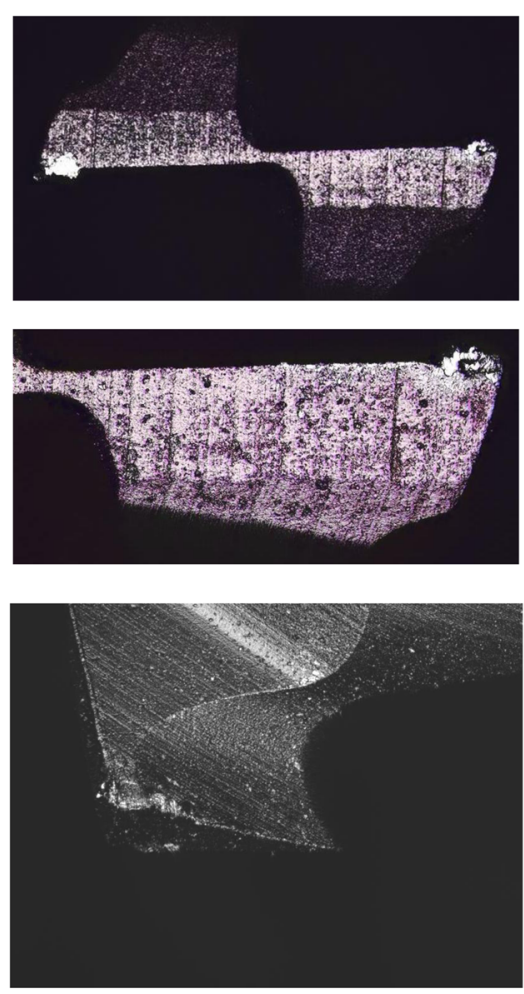

(a)
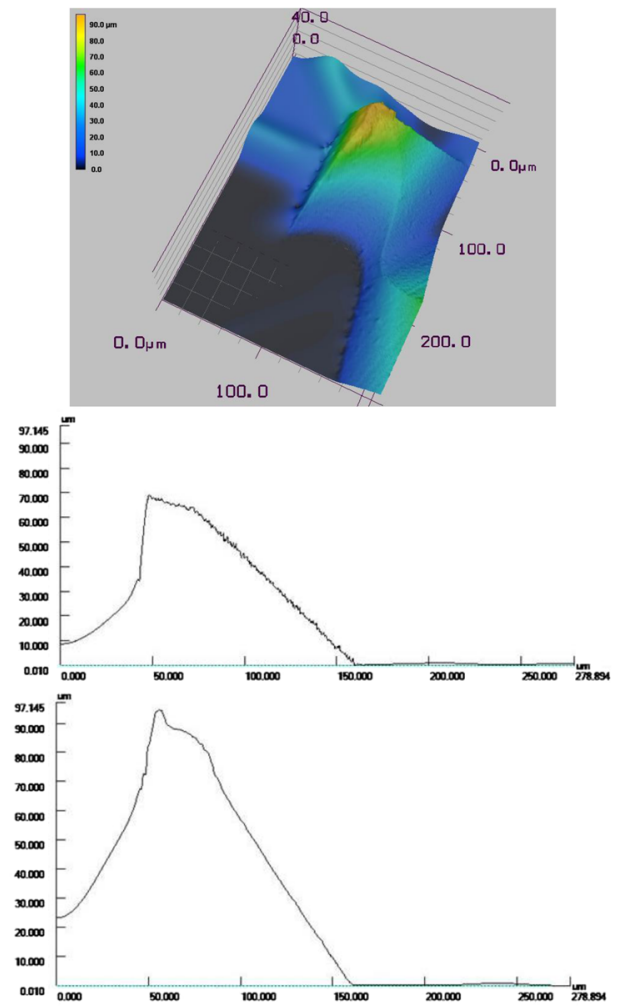

(b)
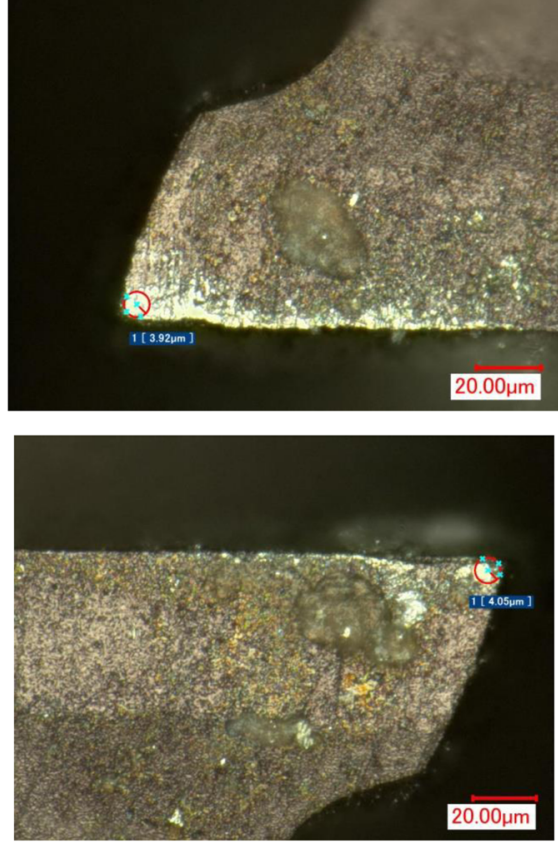

(c)

Fig. 3 a Edge condition of the micro end mill after machining test. b Edge profile of the cutting tool with and without BUE. $\mathbf{c}$ Edge condition of the micro end mill after BUE was removed

following process steps were considered in the analysis. First, the tilt (slope) of the surface was corrected. Second, the noise in the surface height information was removed by applying a denoising algorithm. Figure 5 shows the raw images of the micromilled surfaces for all feed values from the smallest, $0.4 \mu \mathrm{m} /$ tooth (a), to the largest feed, $4 \mu \mathrm{m} /$ tooth (i), based on the experimental plan given in Table 1. Figure 6 shows extracted microscope images of the machined surfaces (with $\times 500$ magnification). The influence of machining conditions is reflected on the micromilled surface. The small material particles from BUE were smeared on the surface. These appear as stochastically distributed hills over the surface. As the feed increases, feed marks become more visible.
Arithmetic mean surface roughness (Sa), surface skewness (Ssk), and surface kurtosis (Sku) parameters are investigated here. Ssk is defined as the ratio of the mean of the height values cubed and the cube of root-mean-square height $(\mathrm{Sq})$ within a sampling area [22]. It describes the shape of the topography height distribution. If Ssk $>0$, then the peaks are dominant on the surface whereas Ssk $<0$ indicates dominance of valleys. Sku is calculated as the ratio of the mean of the fourth power of the height values and the fourth power of Sq within the sampling area [22]. It is a measure of the sharpness of the surface height distribution, and it is strictly positive. Sku $>3.0$ indicates the existence of high peaks or deep valleys on the surface. For a surface representing normal distribution, Ssk is 0 and Sku is 3.
Fig. 4 Two possible configurations of microscale cutting. a Stagnation point assumption. b BUE formation

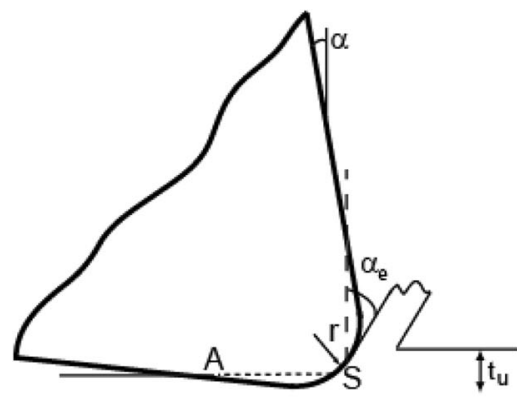

(a)

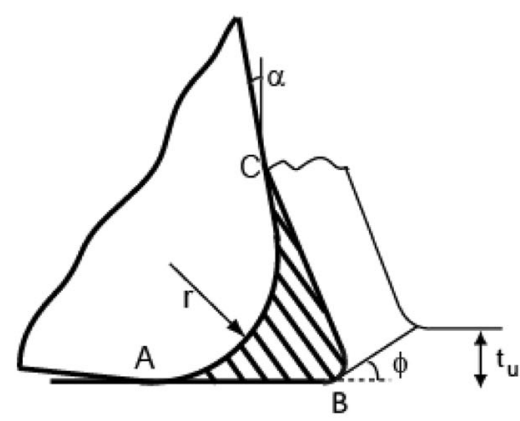

(b) 
Fig. 5 a-i Images of the micromilled surfaces at all feed values

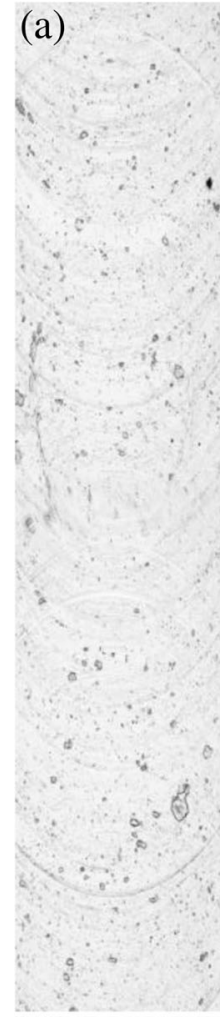

(f)
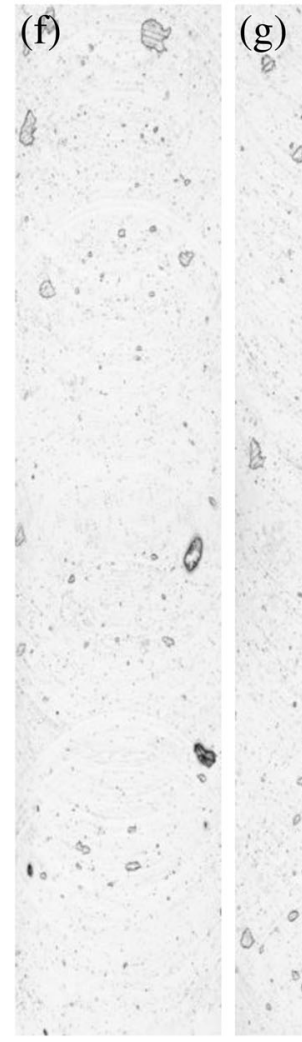

Ssk and Sku were considered to investigate the size and distribution of the rubbed particles on the surface as they represent a histogram of heights which define the symmetry and deviation from an ideal normal distribution. The convention in areal (g)

(b) (c) $\quad$ (d)

(e)

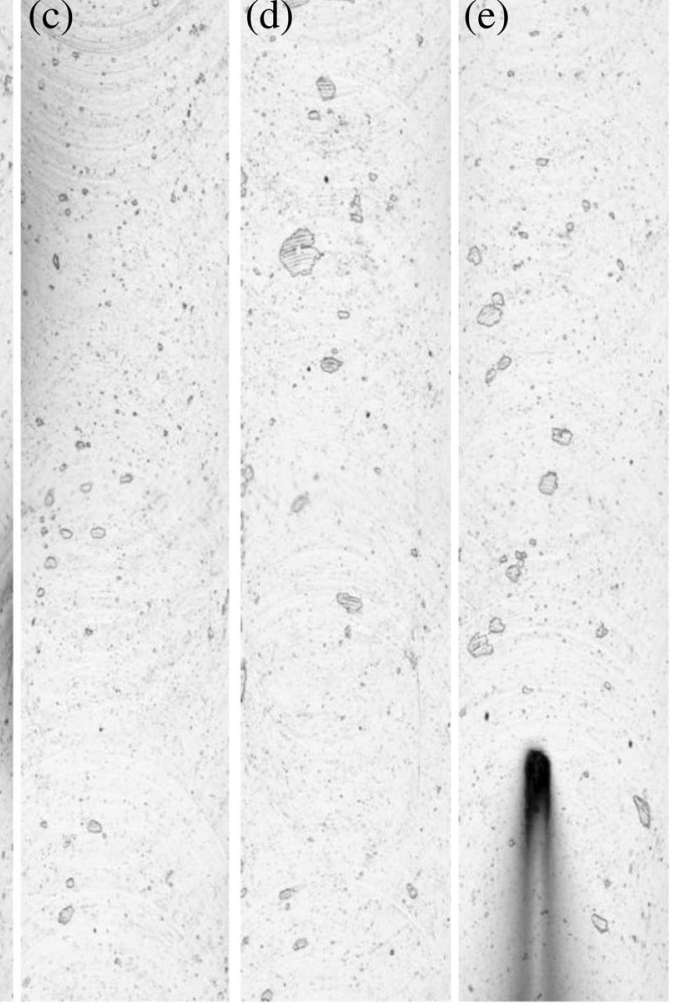

(h)

(i)

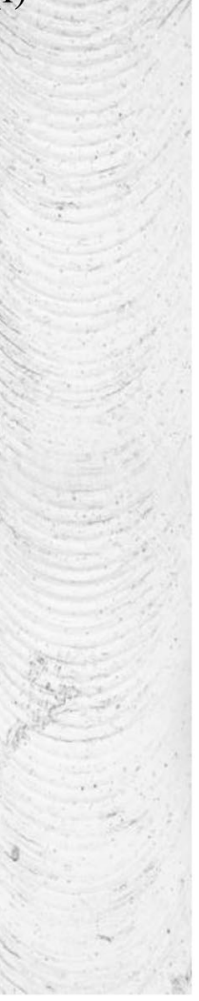

surface texture measurements is to take one sampling area per evolution area [22]. Here, it is decided to divide the surface into nine divisions as shown in Fig. 7, and each division was analyzed separately. This approach allows the investigation of irregular 


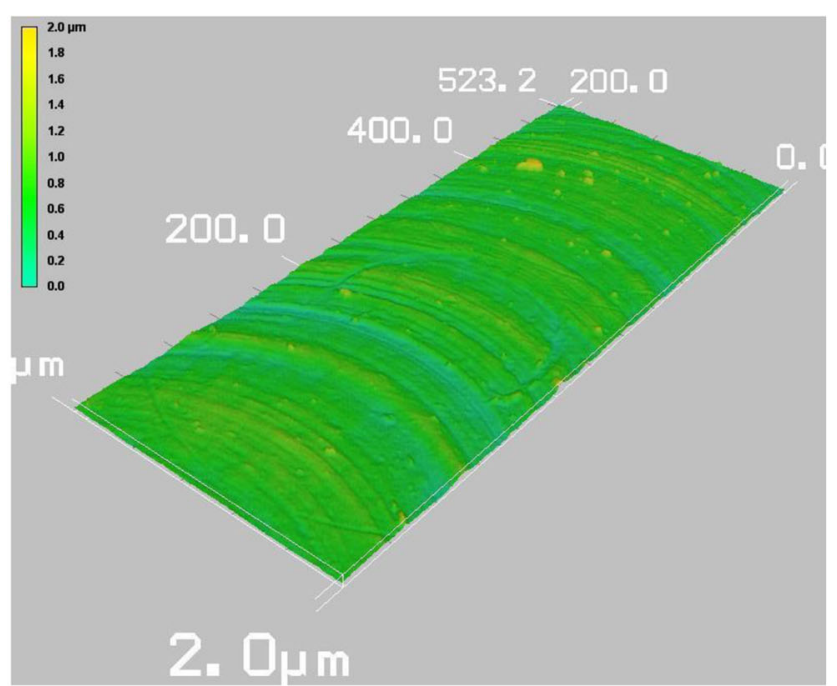

(a)

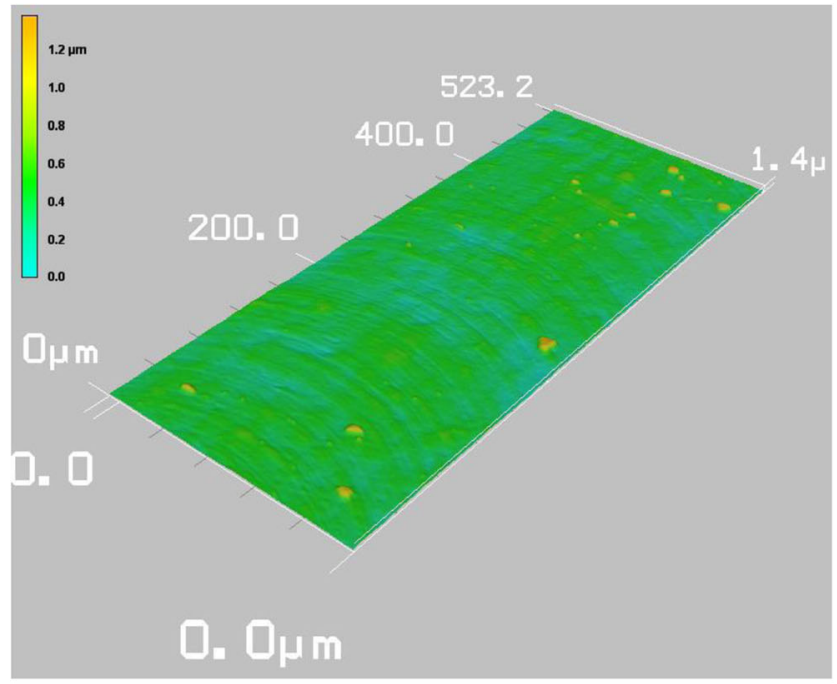

(b)

Fig. 6 Surface topography obtained through confocal laser scanning. a Feed at $0.4 \mu \mathrm{m} /$ tooth. b Feed at $2 \mu \mathrm{m} /$ tooth

surface imperfections in isolation and observation of their variation along the machined surface. The image in Fig. 7a represents an area of $522 \times 210 \mu \mathrm{m}$ obtained by stitching microscope images along the micromilled surface. Figure $7 \mathrm{~b}$ shows the variation in Ssk and Sku at those regions indicated in Fig. 7a. Larger particles in regions 1, 2, and 5 resulted in large values of Ssk and Sku. Large values of Ssk and Sku are due to high-order terms in their equations (Fig. 8).

The trends of the results are in agreement with the force measurements. The lowest surface roughness value of $0.06 \mu \mathrm{m}$ was measured around 1.5-2 $\mu \mathrm{m} /$ tooth, which belongs to the shearing-dominated machining region. As the feed increases further, surface roughness also increases, as expected. However, Ssk and Sku both decrease. A larger and more stable BUE with increasing feed results in less material particles smeared on the machined surface. Increasing the feed further leaves larger

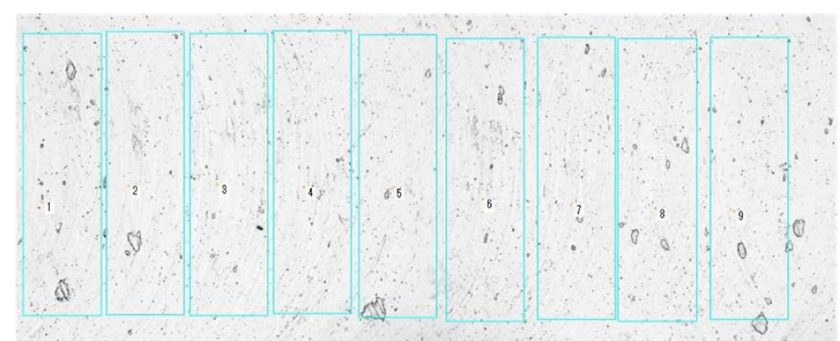

(a)

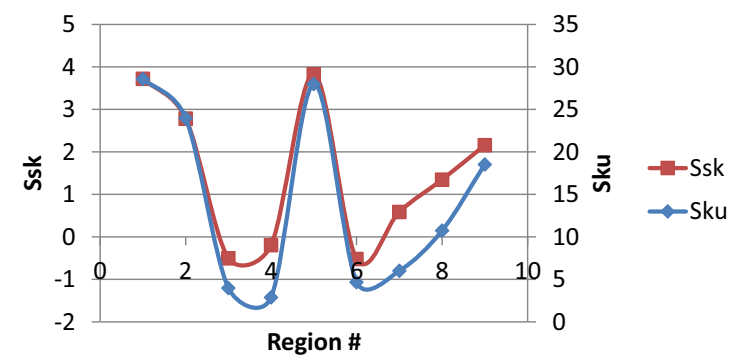

(b)

Fig. 7 a Micromilled surface for $2 \mu \mathrm{m} /$ tooth feed divided into nine regions. b Variation in skewness and kurtosis among regions

surface marks, which increase the areal surface roughness value as seen in Fig. 5h, i. At feed values corresponding to the ploughing and transition regions, Ssk and Sku values increase significantly, indicating the detrimental effect of BUE on the surface texture. Results indicate the importance of feed selection in micromilling and the trade-off between surface texture parameters. With increasing feed, the process behaves like a macroscale milling process where the feed is the most influential parameter on surface roughness. The results correlate with the findings of Jaffery et al. [15]. Recently, Wang et al. [23] showed that peaks on the surface due to BUE hinder the ability to predict surface quality in micromilling.

\section{Micromilling mechanistic force modeling}

In micromilling, typical feed values are in the same order of magnitude as the cutting edge radius. If the uncut chip thickness is less than a certain value (also known as the minimum uncut chip thickness), then the machining operation cannot be performed effectively. As a result, the round cutting edge ploughs the uncut material onto the work surface. Malekian et al. [5] proposed a mechanistic micromilling force model by considering shearing and ploughing phases separately. They were able to simulate dynamic machining forces for the micromilling of aluminum 6061. Ploughing forces were modeled to be proportional to the volume of the material elastically deformed underneath the cutting edge. The amount of elastic recovery, the minimum uncut chip thickness value, the edge radius of the cutting edge, and the clearance angle are the inputs to model ploughing forces. The minimum chip 


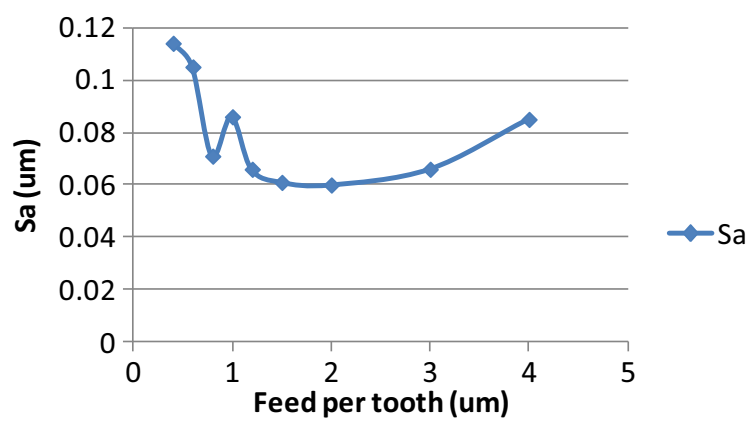

(a)

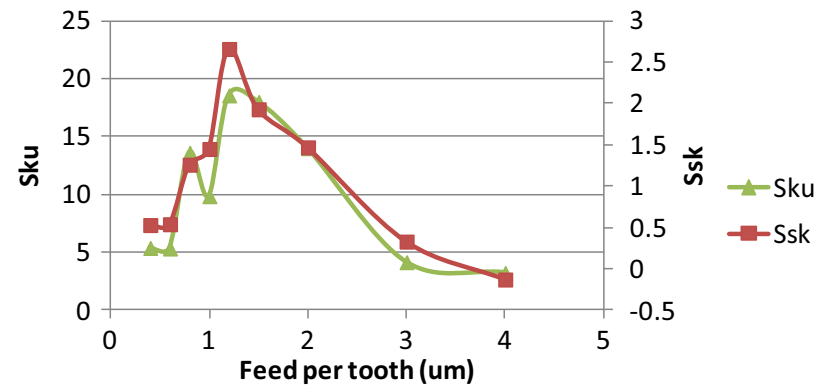

(b)

Fig. 8 a Areal surface roughness (Sa). b Surface skewness (Ssk) and kurtosis (Sku) measurements as a function of feed

thickness is usually defined as the ratio of uncut chip thickness to cutting edge radius, and it is considered to be around $20 \%$ based on the assumption that a stagnation point exists on the cutting edge, which separates the work material flow into the chip and onto the workpiece. In a recent study, Oliaei and Karpat [21] showed that built-up edge formation in front of the cutting edge reduces this ratio down to $10 \%$ during microturning of titanium alloy Ti6Al4V.

Figure 9 shows the micromilling process model proposed by Malekian et al. [5]. The forces acting on the cutting edge are defined with respect to the uncut chip thickness $(h)$. When the uncut chip thickness is larger than a critical value $\left(h>h_{\mathrm{c}}\right)$, shearing dominates the machining process. The tangential and radial forces acting on the cutting edge can be represented with Eq. 1 .

$d F_{\mathrm{t}_{j}}=\left(K_{\mathrm{te}}+K_{\mathrm{tc}} h_{j}\right) d z$

$d F_{\mathrm{r}_{j}}=\left(K_{\mathrm{re}}+K_{\mathrm{rc}} h_{j}\right) d z$

Equation 1 represents the shearing-dominated region, where $K_{\mathrm{rc}}$ and $K_{\mathrm{tc}}$ are defined as the radial and tangential cutting coefficients, respectively. $K_{\mathrm{re}}$ and $K_{\mathrm{te}}$ are the radial and tangential edge coefficients, respectively. These coefficients represent the resistance of the material to machining and the influence of the cutting edge radius. These unknown coefficients are usually calculated based on average machining forces obtained through micromilling experiments. In Eq. $1, h_{j}$ denotes the uncut chip thickness corresponding to cutting edge $j$. The circular tool path assumption in which the uncut chip thickness varies from zero to

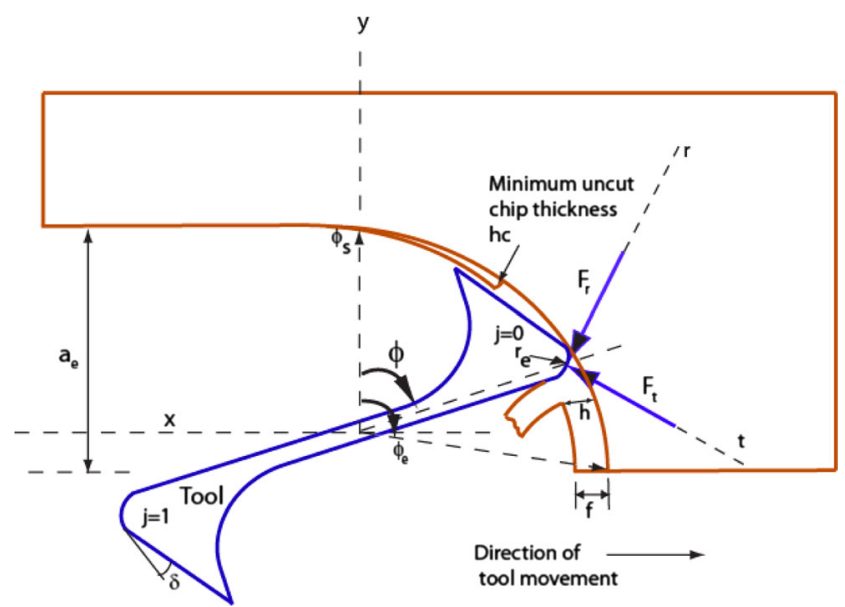

Fig. 9 Micromilling process model

the maximum value of feed per tooth is no longer acceptable in micromilling when tool run-out and feed values are close to each other. As a result, cutting edges of the micro end mills do not experience the same chip load during milling operation, which leads to fluctuations in the machining forces from one cutting edge to another. Tool run-out is directly related to the microtool, the tool holder, and the high-speed spindle. The tool run-out model of Zhang et al. [6] has been adopted in this study. The uncut chip thickness is also a function of the immersion angle and helix angle of the tool acting on a differential height element $(d z)$ on the tool body. As for the region where $h<h_{\mathrm{c}}$, ploughing condition dominates the machining forces. Malekian et al. [5] modeled these forces proportional to the volume of interference between the tool and the workpiece. The ploughing volume regarding a discretized disk element of a tooth is related to the area underneath the tool as $V_{\mathrm{p}}=A_{\mathrm{p}} \cdot d z$. Ploughing forces in radial and tangential directions can be expressed as in Eq. 2.

$d F_{\mathrm{tp}_{j}}=\left(K_{\mathrm{te}}+K_{\mathrm{tp}} A_{\mathrm{p}}\right) d z$
$d F_{\mathrm{rp}_{j}}=\left(K_{\mathrm{re}}+K_{\mathrm{rp}} A_{\mathrm{p}}\right) d z$

$F_{\mathrm{rp}_{j}}$ and $F_{\mathrm{tp}_{j}}$ are the radial and tangential ploughing forces acting on tooth $j$, respectively. Edge coefficients $K_{\mathrm{re}}$ and $K_{\mathrm{te}}$ in the shearing-dominant regime are incorporated together with additional ploughing coefficients based on the ploughed area underneath the cutting edge $K_{\mathrm{rp}}$ and $K_{\mathrm{tp}}$. The estimation procedure of the ploughed area $A_{\mathrm{p}}$ is given in [5]. Equation 3 summarizes the calculation of radial and tangential forces acting on a discretized disk element on tooth $j$ as

$d F_{\mathrm{t}_{j}}=\left\{\begin{array}{lc}\left(K_{\mathrm{te}}+K_{\mathrm{tc}} h_{j}\right) d z & \text { when } h \geq h_{\mathrm{c}} \\ \left(K_{\mathrm{te}}+K_{\mathrm{tp}} A_{\mathrm{p}}\right) d z & \text { when } h<h_{\mathrm{c}}\end{array}\right\}$
$d F_{\mathrm{r}_{j}}=\left\{\begin{array}{cc}\left(K_{\mathrm{re}}+K_{\mathrm{rc}} h_{j}\right) d z & \text { when } h \geq h_{\mathrm{c}} \\ \left(K_{\mathrm{re}}+K_{\mathrm{rp}} A_{\mathrm{p}}\right) d z & \text { when } h<h_{\mathrm{c}}\end{array}\right\}$ 
A coordinate transformation is then required to calculate the forces in $\mathrm{X}$ and $\mathrm{Y}$ directions. For measured micromilling forces in $\mathrm{X}$ and $\mathrm{Y}$ directions, it is possible to identify unknown coefficients in reverse fashion.

$d F_{\mathrm{x}_{j}}=d F_{\mathrm{t}_{j}} \cos \varphi+d F_{\mathrm{r}_{j}} \sin \varphi$

$d F_{\mathrm{y}_{j}}=d F_{\mathrm{t}_{j}} \sin \varphi-d F_{\mathrm{r}_{j}} \cos \varphi$

Cutting, edge, and ploughing force coefficients shown in Eq. 3 together with tool run-out parameters (its magnitude and angle) can be calculated based on a methodology proposed by Malekian et al. [5]. There are a total of eight unknowns (six force coefficients and two tool run-out parameters) that need to be identified. For simplification, it can be assumed that the tool run-out magnitude and angle are the same for all feed values. The objective function can be written as Eq. 5, which is the sum of the squared error between the data points of the measured cutting forces and the force predictions, where $F_{\text {measured }}$ is the instantaneous experimental cutting force data point and $F_{\text {predicted }}$ corresponds to the prediction, $l$ is the number of feed values, and $k$ is the number of data points.

error $=\sum_{i=1}^{k} \sum_{j=1}^{l}\left(F_{\text {measured }_{i, j}}-F_{\text {predicted }_{i, j}}\right)^{2}$

The goal of this study is to calculate the distributions of force coefficients using Bayesian inference, which is explained in the next section.

\section{Bayesian inference}

Bayes' rule provides a rational method for updating beliefs in light of new information (i.e., experimental measurements). It is represented with Eq. 6 .

$$
\{A \mid B, \theta\}=\frac{\{A \mid \theta\}\{B \mid A, \theta\}}{\{B \mid \theta\}}
$$

The left-hand side of the equation $\{A \mid B, \theta\}$ is the posterior distribution, which summarizes the state of knowledge about an event $A$ in a statistical model, after observing the result $B$. The first term on the right-hand side of the equation $\{A \mid \theta\}$ is the prior distribution about an uncertain event $A$, at a state of information $\theta$, which addresses our state of knowledge about the parameters before having an observation. The second term $\{B \mid A, \theta\}$ is the likelihood of obtaining an experimental result $B$ given that the observation $A$ has occurred. The denominator $\{B \mid \theta\}$ is the probability of obtaining an experimental result $B$ without knowing that $A$ has occurred, which behaves like a normalizing constant. Usually, the denominator is not computed explicitly, as it is known that the posterior distribution is a probability density function that integrates to one. According to Bayes' rule, the posterior belief is proportional to multiplication of prior and likelihood functions. This process of learning via Bayes' rule is referred to as Bayesian inference, i.e., updating prior beliefs given new data $B$ to obtain the posterior belief.

Karandikar et al. [9] proposed the formulation of updating the force coefficients, given the measured values of experimental force data. The variability of the force coefficients can be assessed by combining prior knowledge and experimental data. Bayes' rule for the force coefficients, including ploughing coefficients, could be written as

$$
\begin{aligned}
& f_{K_{\mathrm{tc}}, K_{\mathrm{rc}}, K_{\mathrm{te}}, K_{\mathrm{re}}, K_{\mathrm{tp}}, K_{\mathrm{rp}}}\left(K_{\mathrm{tc}}, K_{\mathrm{rc}}, K_{\mathrm{te}}, K_{\mathrm{re}}, K_{\mathrm{tp}}, K_{\mathrm{rp}} \mid \bar{F}_{\mathrm{x}, \mathrm{m}}, \bar{F}_{\mathrm{y}, \mathrm{m}}\right) \alpha \\
& f_{K_{\mathrm{tc}}, K_{\mathrm{rc}}, K_{\mathrm{te}}, K_{\mathrm{re}}, K_{\mathrm{tp}}, K_{\mathrm{rp}}} l\left(\bar{F}_{\mathrm{x}, \mathrm{m}}, \bar{F}_{\mathrm{y}, \mathrm{m}} \mid K_{\mathrm{tc}}, K_{\mathrm{rc}}, K_{\mathrm{te}}, K_{\mathrm{re}}, K_{\mathrm{tp}}, K_{\mathrm{rp}}\right)
\end{aligned}
$$

In Eq. 7, $\bar{F}_{\mathrm{x}, \mathrm{m}}$ and $\bar{F}_{\mathrm{y}, \mathrm{m}}$ are the measured mean forces in $x$ and $y$ directions, respectively. The term $f_{K_{\mathrm{tc}}, K_{\mathrm{re}}, K_{\mathrm{te}}, K_{\mathrm{re}}, K_{\mathrm{tp}}, K_{\mathrm{rp}}}$ $\left(K_{\mathrm{tc}}, K_{\mathrm{rc}}, K_{\mathrm{te}}, K_{\mathrm{re}}, K_{\mathrm{tp}}, K_{\mathrm{rp}} \mid \bar{F}_{\mathrm{x}, \mathrm{m}}, \bar{F}_{\mathrm{y}, \mathrm{m}}\right)$ is the posterior distribution of the force coefficients given measured values of the mean forces. The term $f_{K_{\mathrm{tc}}, K_{\mathrm{rc}}, K_{\mathrm{te}}, K_{\mathrm{re}}, K_{\mathrm{tp}}, K_{\mathrm{rp}}}$ is the joint prior distribution of the force coefficients, and $l$ $\left(\bar{F}_{\mathrm{x}, \mathrm{m}}, \bar{F}_{\mathrm{y}, \mathrm{m}} \mid K_{\mathrm{tc}}, K_{\mathrm{rc}}, K_{\mathrm{te}}, K_{\mathrm{re}}, K_{\mathrm{tp}}, K_{\mathrm{rp}}\right)$ is the likelihood of obtaining mean forces in the $x$ and $y$ directions, given the six force coefficients. The multiplication of prior and likelihood is proportional to the posterior function, which allows for updating beliefs, and then a normalization must be made as in Eq. 6. Assuming that the force coefficients are independent, the density function of the joint force coefficients $f_{K_{\mathrm{tc}}, K_{\mathrm{rc}}, K_{\mathrm{te}}, K_{\mathrm{re}}, K_{\mathrm{tp}}, K_{\mathrm{rp}}}$ is equal to the multiplication of the density function of each force coefficient. The mean forces in $x$ and $y$ directions $\left(\bar{F}_{\mathrm{x}, \mathrm{m}}, \bar{F}_{\mathrm{y}, \mathrm{m}}\right)$ are also assumed to be independent; thus, the likelihood of obtaining mean force measurements is multiplied for each direction in order to obtain a joint likelihood function.

In order to carry out the Bayesian updating procedure, two main inputs are required; the likelihood and prior functions. The prior function corresponds to our prior knowledge on specific force coefficients, and the likelihood function refers to the likelihood of obtaining the experimental mean force values, given specified values of the force coefficients. To select these probability distributions, a well-known bellshaped normal distribution might be an appropriate choice. In that case, it is assumed that force coefficients are distributed symmetrically around a known mean. The other option is to use non-informative, in other words, uniform priors to capture the pattern with minimal knowledge. This type of selection is favorable for situations with insufficient previous evidence or 
expertise, or when obtaining the related information requires tedious research. In this study, both uniform and normal prior distributions were employed. In order to define a normal distribution, mean and standard deviation are required as parameters. On the other hand, minimum and maximum values are required to define a uniform distribution.

\subsection{Markov chain Monte Carlo method}

The MCMC method is used to draw samples from a random known distribution. The Metropolis-Hastings (MH) algorithm is one of the most popular MCMC methods, and it is primarily used as a way to simulate observations from unwieldy distributions [17]. Therefore, the MH method can be used for drawing samples from a random known distribution, which, in our case, is the posterior distribution force coefficients [18]. In the $\mathrm{MH}$ algorithm, the proposal distribution denoted by $q(x)$ is used to draw candidate samples that mimic samples drawn from the target distribution denoted by $p(x)$. The candidate samples from the proposal distribution are either accepted or rejected depending on an acceptance probability given below:

$A\left(x, x^{*}\right)=\min \left\{1, \frac{p\left(x^{*}\right) q\left(x \mid x^{*}\right)}{p(x) q\left(x^{*} \mid x\right)}\right\}$

where $x *$ is the candidate sample drawn from a proposal distribution $q(x)$ and $x$ is the current state of the Markov chain. For each iteration, the Markov chain moves to $x *$ if the sample is accepted; otherwise, the chain stays on the current value of $x$. The pseudo-code of the MH algorithm is shown below:

1. Initialize the starting point $x 0$

2. For $i=0$ to $i=N-1$ iterations, do the following:

a) Sample $x^{0} \sim q\left(x^{*} \mid x^{i}\right)$

b) Sample $u \sim U_{[0,1]}$

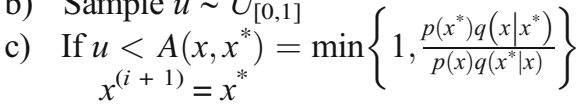

else

$$
x^{(i+1)}=x^{(i)}
$$

The MH algorithm has been carried out to approximate posterior distribution of force coefficients. Since the posterior distribution we want to approximate is a joint density function of force coefficients, sampling for each variable is carried out using univariate proposal distributions. One variable at a time is sampled, and then sequentially, the algorithm proceeds to the remaining variables. To illustrate the application of $\mathrm{MH}$ algorithm:

1. The initial values for all force coefficients are conducted as the first step.
Table 2 Identified force coefficients and run-out parameters after optimization

\begin{tabular}{ll}
$K_{\text {te }}\left(\mathrm{N} / \mathrm{mm}^{2}\right)$ & 9.1 \\
$K_{\text {tc }}\left(\mathrm{N} / \mathrm{mm}^{2}\right)$ & 4475 \\
$K_{\text {re }}\left(\mathrm{N} / \mathrm{mm}^{2}\right)$ & 13.2 \\
$K_{\text {rc }}\left(\mathrm{N} / \mathrm{mm}^{2}\right)$ & 2854 \\
Run-out magnitude $(\mu \mathrm{m})$ & 0.19 \\
Run-out angle $\left({ }^{\circ}\right)$ & 101 \\
$K_{\text {tp }}\left(\mathrm{kN} / \mathrm{mm}^{3}\right)$ & 13,623 \\
$K_{\text {rp }}\left(\mathrm{kN} / \mathrm{mm}^{3}\right)$ & 4300 \\
\hline
\end{tabular}

2. The proposal distributions for each force coefficient $K$ are defined. Proposal distributions have a significant effect on the convergence process. If we sample a wider range of $K$ 's, the proportion of the rejection will probably increase, and therefore, convergence could not be done, or requires significant numbers of iteration. On the other hand, if we select them too narrow, most of the samples will be accepted and the space could not be explored. The key point for selecting proposal distributions is that the range of samples obtained from proposal distributions should include the range of target distributions. It is highlighted that a $25-35 \%$ acceptance rate is appropriate for the convergence of the Markov chain [19].

3. A function needs to be defined in order to estimate force averages given a set of $K$ 's. Since experimental forces are close to zero and tool run-out is dominant in micromilling, using analytic formulas to estimate average forces results in additional errors as they underestimate, or overestimate, the effect of tool run-out on the mean forces. In this research, instead of employing analytic formulas to estimate average force equations, time-domain simulations with tool run-out extension were conducted and their averages were taken into account.

4. The acceptance ratio defined in Eq. 8 should be estimated by an operator which needs to be defined. This operator takes the candidate and current values of the chain as input, evaluates the probabilities according to the prior and likelihood functions, and finally outputs the acceptance probability.

5. For each iteration, one variable at a time is sampled. So for every variable, basically, we have two sets of $K$ values, first is the current set of $K$ 's in state $i$, which is $\left[K_{1}^{i}, K_{1}^{i}\right.$, $\left.K_{1}^{i}, \ldots, K_{n}^{i}\right]$, and the candidate set, $\left[K_{1}^{*}, K_{1}^{i}, K_{1}^{i}, \ldots, K_{n}^{i}\right]$. Besides these sets, force averages are estimated using both sets and taken as inputs to the acceptance ratio operator. Then, the acceptance ratio is estimated and compared with $u$, which is a random number generated from a uniform distribution with a range from 0 to 1 . According to the comparison, $K_{1}^{*}$ is either accepted or rejected. If it is accepted, $K_{1}^{i}$ is updated to $K_{1}^{*}$. The same procedure is repeated continually for the next variable until the sample from $K_{n}^{i}$ is drawn and evaluated. Finally, the iteration ends and the next iteration $i+1$ takes place. 
Table 3 Parameters of prior distributions for uniform and normal settings

\begin{tabular}{llllll}
\hline & \multicolumn{2}{l}{ Normal distribution } & & \multicolumn{2}{l}{ Uniform distribution } \\
\cline { 2 - 3 } \cline { 5 - 6 } & Mean & Standard deviation & & Lower & Upper \\
\hline$K_{\mathrm{te}}(\mathrm{N} / \mathrm{mm})$ & 9.1 & 3 & 0 & 25 \\
$K_{\mathrm{tc}}\left(\mathrm{N} / \mathrm{mm}^{2}\right)$ & 4475 & 300 & 0 & 10,000 \\
$K_{\mathrm{re}}\left(\mathrm{N} / \mathrm{mm}^{2}\right)$ & 13.2 & 3 & 0 & 25 \\
$K_{\mathrm{rc}}\left(\mathrm{N} / \mathrm{mm}^{2}\right)$ & 2854 & 300 & 0 & 10,000 \\
$K_{\mathrm{tp}}\left(\mathrm{kN} / \mathrm{mm}^{3}\right)$ & 13,623 & 1400 & 0 & 30,000 \\
$K_{\mathrm{rp}}\left(\mathrm{kN} / \mathrm{mm}^{3}\right)$ & 4307 & 400 & 0 & 10,000 \\
\hline
\end{tabular}

6. When the iterations end, we obtain samples from the distributions of $K$ 's and the traces of them. The burn-in

Fig. 10 Traces (a) and sampled force coefficients (b) of force coefficients for normal prior setting

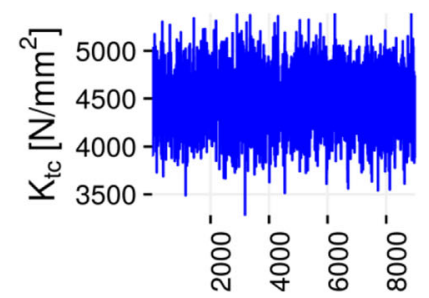

\# of iterations
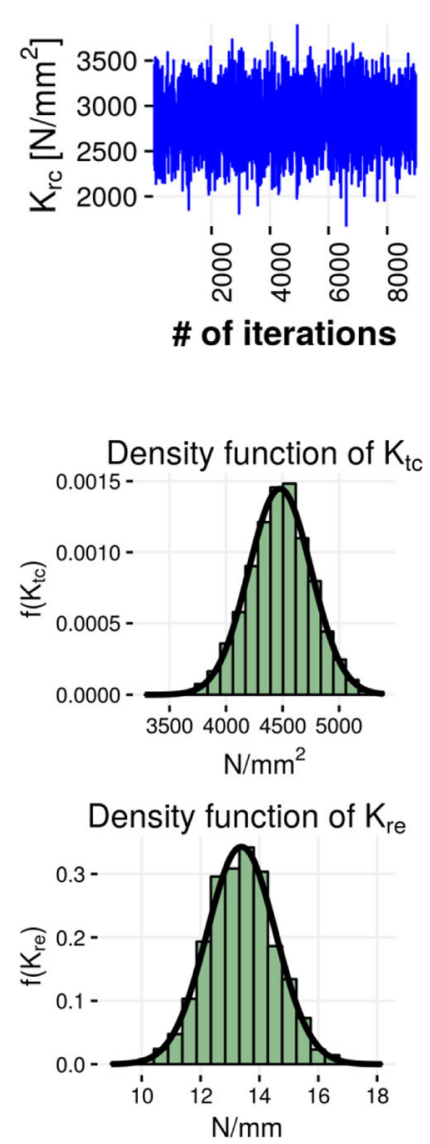

process is applied, which refers to discarding an initial portion of the simulation in order to ensure steady-state conditions. Finally, normal distributions are fit to the samples. The maximum-likelihood values for the mean and standard deviation of the normal distribution correspond to sample statistics for the data.

\subsection{Bayesian inference applied to milling force modeling}

Ploughing forces are modeled to be proportional to the volume of the elastically deformed material underneath the cutting edge which is defined by those variables. Several variables such as clearance angle, edge radius, elastic recovery, and critical chip thickness must be known to calculate ploughing forces.
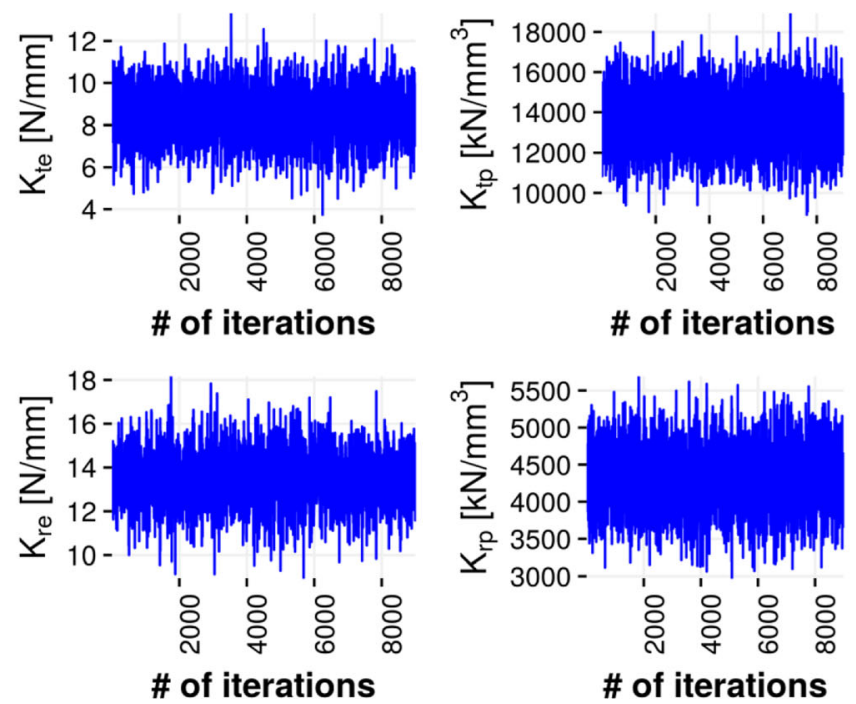

(a)
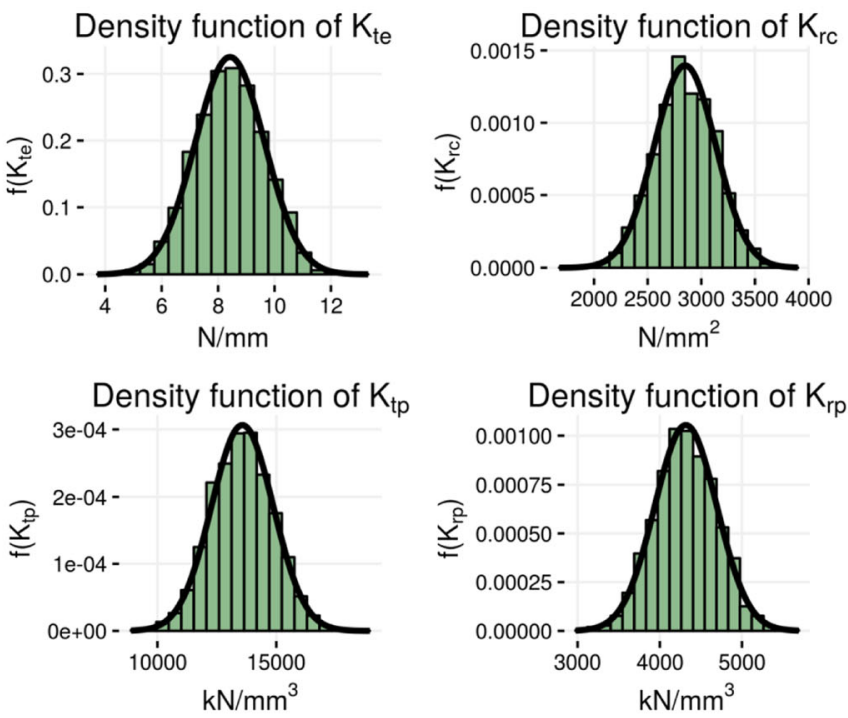

(b)

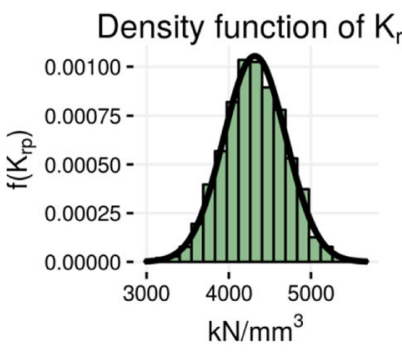


Fig. 11 Posterior (blue lines) and prior (red dashed lines) distributions of the force coefficients. a Normal. b Uniform
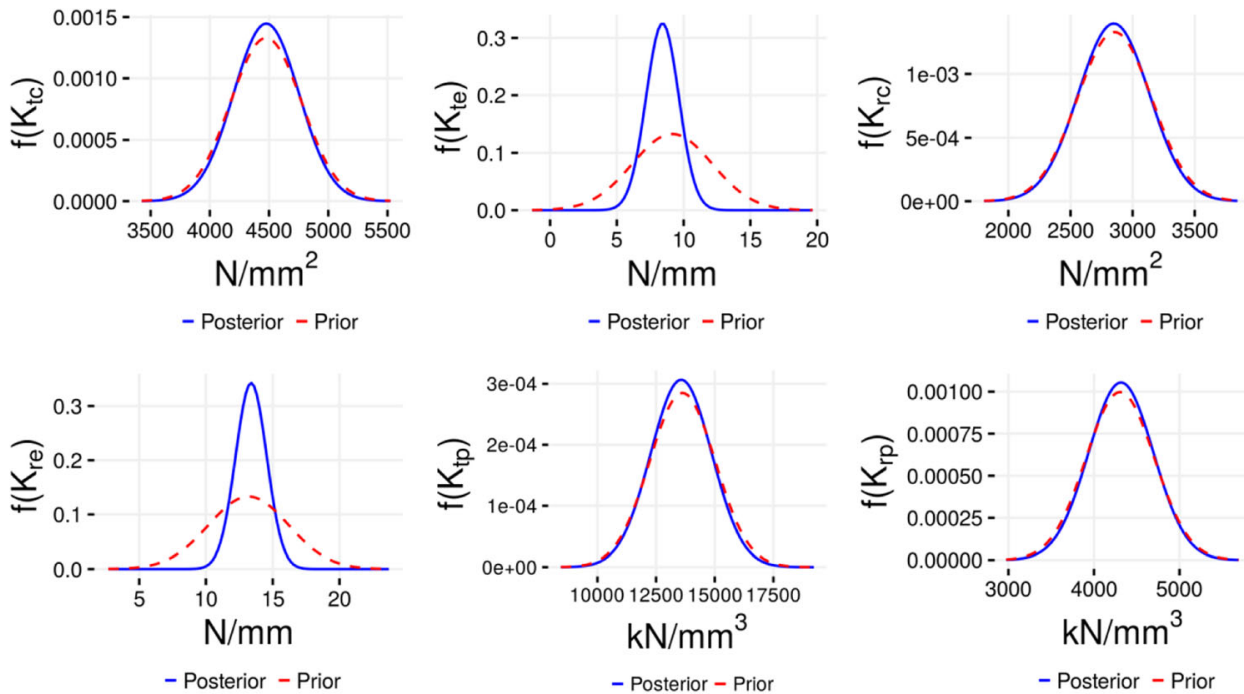

(a)
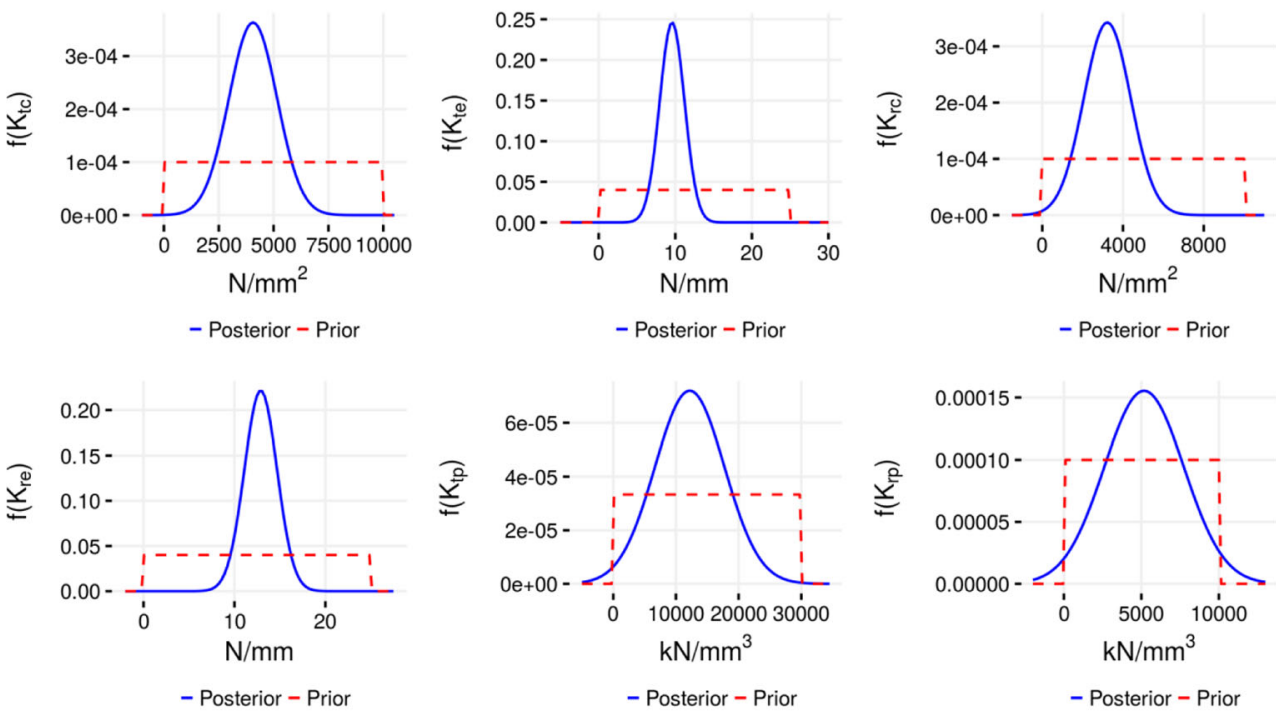

Identified ploughing force coefficients $K_{\mathrm{tp}}$ and $K_{\mathrm{rp}}$ depend on the condition of the tool edge as described in Fig. 4. Assuming that

Table 4 Parameters of obtained posterior distributions for uniform and normal settings

\begin{tabular}{llllll}
\hline & \multicolumn{2}{l}{ Normal distribution } & & \multicolumn{2}{l}{ Uniform distribution } \\
\cline { 2 - 3 } \cline { 5 - 6 } \cline { 5 - 5 } & Mean & Standard deviation & & Mean & Standard deviation \\
\hline$K_{\text {te }}(\mathrm{N} / \mathrm{mm})$ & 8.4 & 1.2 & 9.6 & 1.6 \\
$K_{\text {tc }}\left(\mathrm{N} / \mathrm{mm}^{2}\right)$ & 4477 & 276 & 4062 & 1098 \\
$K_{\text {re }}\left(\mathrm{N} / \mathrm{mm}^{2}\right)$ & 13.4 & 1.2 & 12.9 & 1.8 \\
$K_{\text {rc }}\left(\mathrm{N} / \mathrm{mm}^{2}\right)$ & 2849 & 286 & 3222 & 1165 \\
$K_{\text {tp }}\left(\mathrm{kN} / \mathrm{mm}^{3}\right)$ & 13,571 & 1300 & 12,195 & 5545 \\
$K_{\text {rp }}\left(\mathrm{kN} / \mathrm{mm}^{3}\right)$ & 4316 & 378 & 5175 & 2566 \\
\hline
\end{tabular}

(b)

BUE acts as a cutting edge, the radius of the cutting edge with BUE was measured as $5 \mu \mathrm{m}$, the clearance angle was measured as $7^{\circ}$, and the elastic recovery percentage is assumed to be $10 \%$. However, considering the dynamic nature of BUE, these variables cannot be identified easily. A larger ploughing area at the tool-work interface would result in lower ploughing force coefficients. It is important to note that uncertainties about these variables are the reason for using the simple force models described in Section 4. The development of detailed models is constrained by the uncertainties of the input parameters.

An open-source software $\mathrm{R}$ was used to calculate unknown coefficients using genetic algorithm. In the solution algorithm, first, cutting and edge coefficients for the shearing region were considered. Two ploughing coefficients $\left(K_{\mathrm{tp}}, K_{\mathrm{rp}}\right)$ were calculated as a second step based on already calculated edge force coefficients. Table 2 shows the calculated force coefficients and run- 


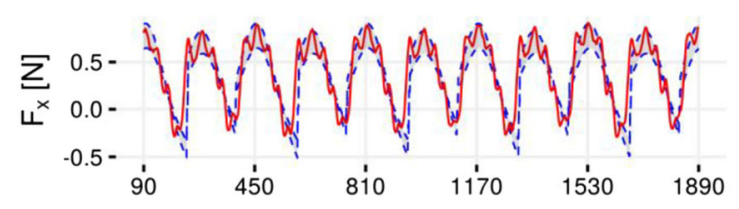

$\varphi$ (deg)

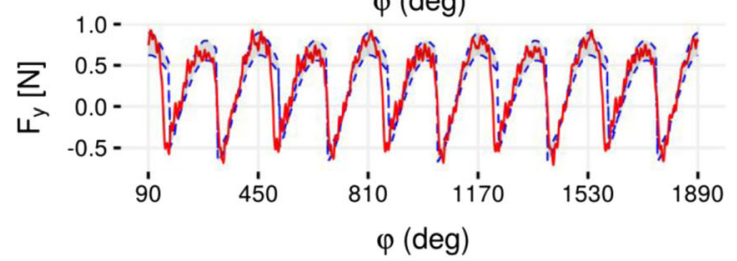

(a)
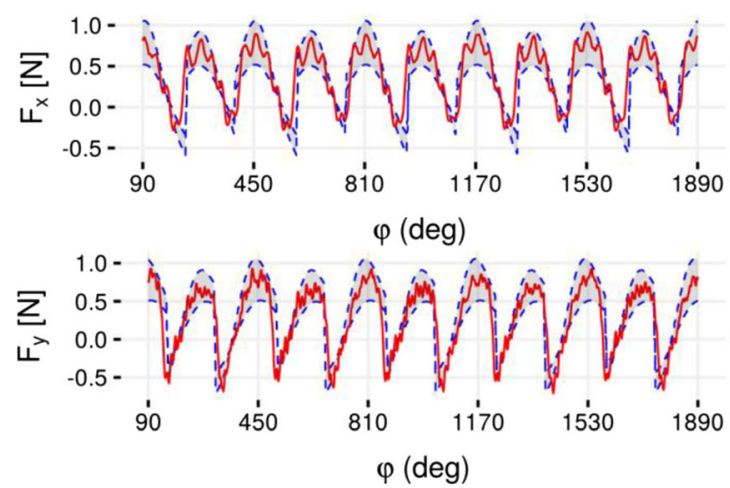

(c)

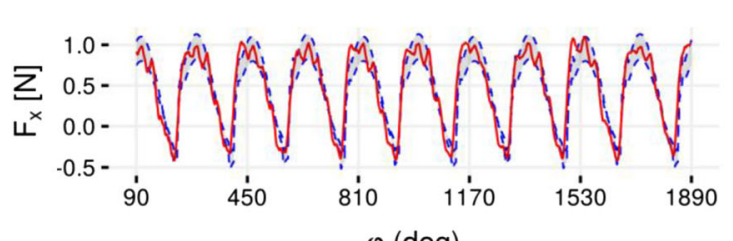

$\varphi($ deg)

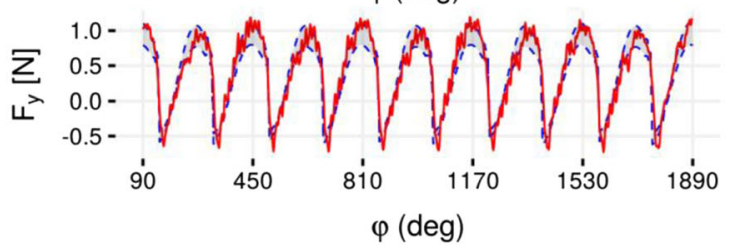

(b)

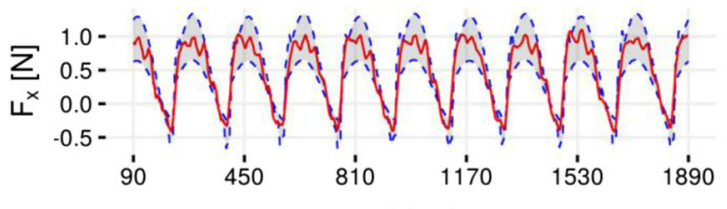

$\varphi($ deg $)$

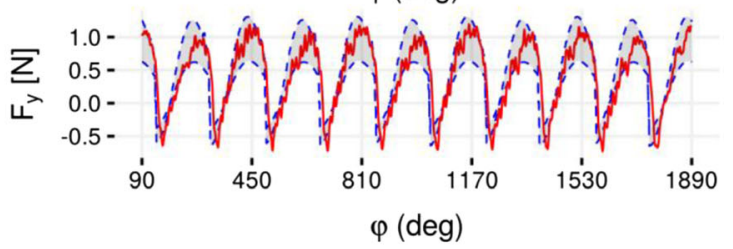

(d)

Fig. 12 Simulated and measured force predictions for a $0.4 \mu \mathrm{m} /$ tooth - normal distribution, b $2 \mu \mathrm{m} /$ tooth $—$ normal distribution, c $0.4 \mu \mathrm{m} /$ toothuniform distribution, and $\mathbf{d} 2 \mu \mathrm{m} /$ tooth - uniform distribution

out parameters which minimize Eq. 5 for all experimental conditions given in Table 1.

Deterministic point estimates of force coefficients obtained from the genetic algorithm approach are selected as means for normal prior distributions. Since many experiments were performed and force measurements were taken, there is enough information about the process. On the other hand, if prior knowledge or experimental data is not available, a uniform prior can also be chosen. Both approaches are used in this study to compare the results. Table 3 shows the selected mean and standard deviation parameters for normal prior distribution and lower and upper values of uniform prior distributions. In uniform distribution, any value is equally likely within the given range.

The likelihood function needs to be addressed in order to employ Bayesian updating. It is assumed that experimental

Table 5 Summary of second set of experiments

\begin{tabular}{llllll}
\hline $\begin{array}{l}\text { Tool } \\
\text { diameter } \\
(\mathrm{mm})\end{array}$ & $\begin{array}{l}\text { Rotational } \\
\text { speed } \\
(\mathrm{rpm})\end{array}$ & $\begin{array}{l}\text { Axial } \\
\text { depth of } \\
\text { cut }(\mu \mathrm{m})\end{array}$ & $\begin{array}{l}\text { Feed per } \\
\text { tooth } \\
(\mu \mathrm{m} / \mathrm{rev})\end{array}$ & $\begin{array}{l}\text { Radial } \\
\text { immersion }\end{array}$ & Type \\
\hline 0.4 & 28,000 & 40 & 2 & $100,60,25$ & $\begin{array}{c}\text { Upmilling, } \\
\text { downmil- } \\
\text { ling }\end{array}$ \\
\hline
\end{tabular}

mean force values distributed normally with a standard deviation of $0.1 \mathrm{~N}$. The mean of the likelihood corresponds to the average experimental force data obtained, as it is an estimate obtained from the slot milling experiments. The likelihood function used in Bayesian learning behaves like an errorcorrecting mechanism, providing a way to overcome problems caused by linear regression. For instance, after experimental force averages are used to update our prior knowledge, the non-linearity of the force averages at small feed values is compensated and the large confidence intervals narrowed for higher feed values. The Metropolis-Hastings algorithm was applied for $10^{4}$ iterations to obtain samples from the joint target densities of force coefficients, and the first 1000 samples were discarded. Figure 10 shows the traces and sampled force coefficients for normal prior. Figure 11 shows the prior and posterior distributions of the force coefficients. Table 4 shows the calculated posterior distribution parameters.

The results shown in Table 4 are close to each other in terms of mean values and close to those shown in Table 2 . The results demonstrate the effectiveness of Bayesian inference even when uniform prior distribution was used. The edge forces in normal prior distribution are affected by the MCMC algorithm more than the cutting force coefficients as seen in Fig. 11a. The variation in uniform posterior distribution parameters is larger than normal distribution. The posterior 


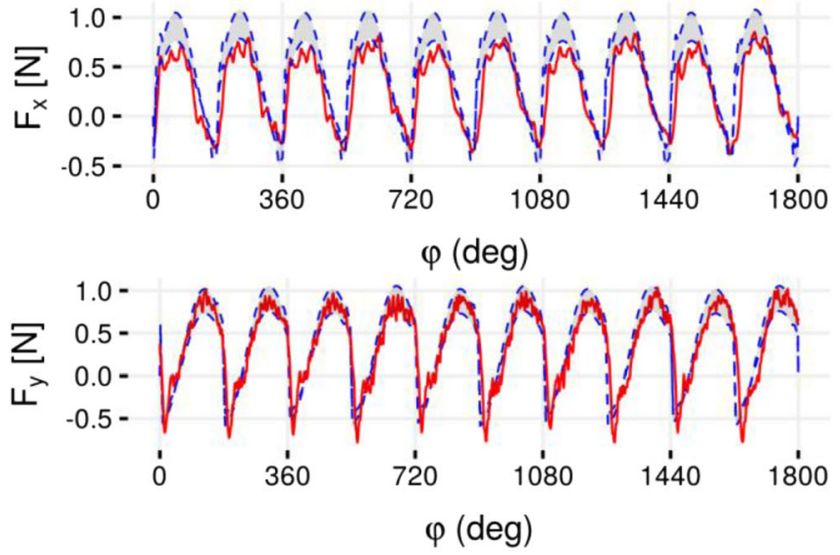

(a)
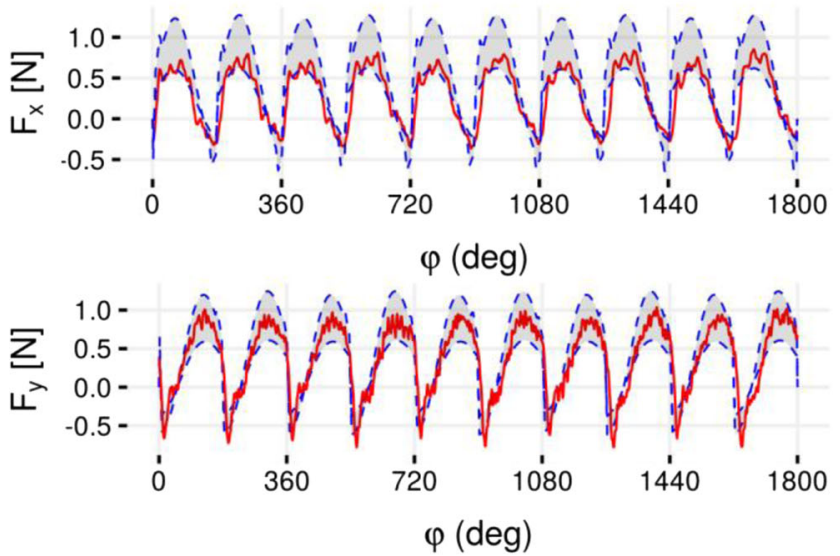

(b)

Fig. 13 Simulated and measured force predictions for $2 \mu \mathrm{m} /$ tooth feed slot milling with a normal distribution and $\mathbf{b}$ uniform prior distribution

distribution is sensitive to the selection of prior distribution. In order to test the predictive ability of these distributions, two cases are considered such as 0.5 and $2 \mu \mathrm{m} /$ tooth feed slot micromilling. The cutting speed and axial depth of cut was kept the same. The upper and lower limits (blue dashed lines) of the force predictions are plotted together according to $95 \%$ confidence interval ( \pm 2 standard deviations) with experimental measurements in Fig. 12. The experimental measurements are observed to be within the predictions. The range of the predictions with uniform distribution is larger. Such predictions can be useful to calculate milling stability boundary calculations including force coefficient uncertainties [24].

\section{Validation of the Bayesian inference model}

In order to test the generalization capability of the developed Bayesian milling model, additional test cases were considered. In the second set of experiments, different test conditions were conducted with a different micro end mill. Table 5 shows the experimental cases where various radial immersion (RI) tests were conducted. Figure 13 shows the model prediction for the $2 \mu \mathrm{m} /$ tooth feed case with both posterior distributions. Both predictions are acceptable in terms of $F_{\mathrm{y}}$ forces. $F_{\mathrm{x}}$ forces are predicted on the lower limit, indicating a difference in tool edge conditions.

Figure 14 shows the experimental measurement of forces with different RI test cases. RI cases of 25 and $60 \%$ are considered in both upmilling and downmilling conditions. With decreasing RI percentage, $F_{\mathrm{y}}$ forces increase while $F_{\mathrm{x}}$ forces decrease in downmilling. In upmilling, forces are lower in magnitude and with decreasing RI, $F_{\mathrm{x}}$ forces increase and $F_{\mathrm{y}}$ forces decrease.

Figure 15 shows the model predictions. While 60\% RI results are acceptable, as the predictions are within the limits, model predictions at $25 \% \mathrm{RI}$ are poor especially for the $F_{\mathrm{y}}$ forces in both upmilling and downmilling tests.

In order to investigate the possible reasons which may cause this difference, cutting edges of the tools after the $25 \%$ immersion tests were investigated as shown in Fig. 16. No
Fig. 14 Measured micromilling forces at $2 \mu \mathrm{m} /$ tooth and axial depth of cut $40 \mu \mathrm{m}$. a $60 \% \mathrm{RI}-$ downmilling. b $25 \% \mathrm{RI}-$ downmilling. c $60 \%$ RIupmilling. d 25\% RI-upmilling
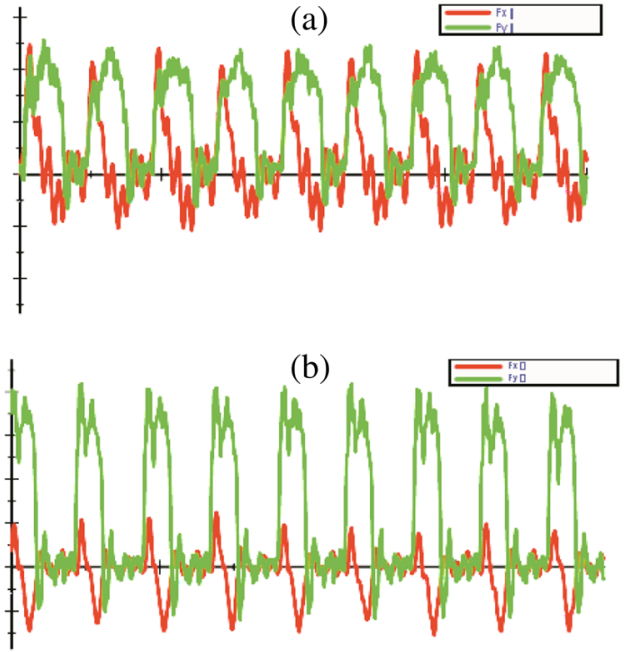
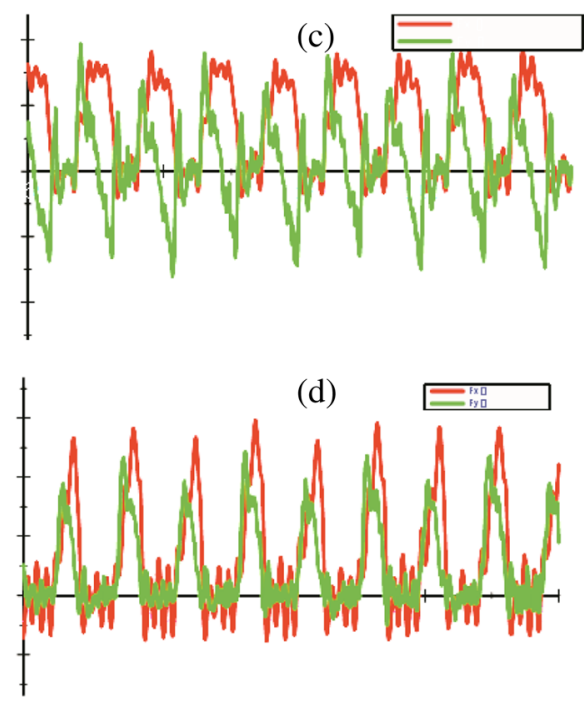

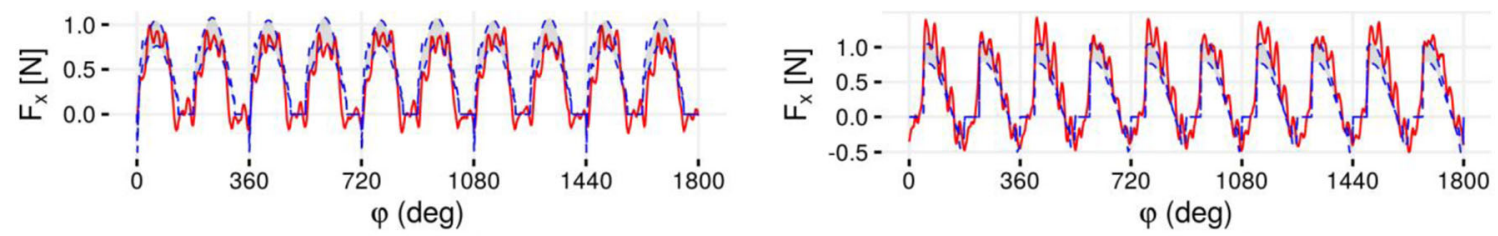

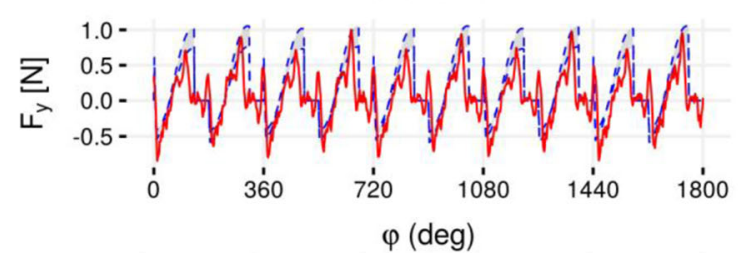

(a)
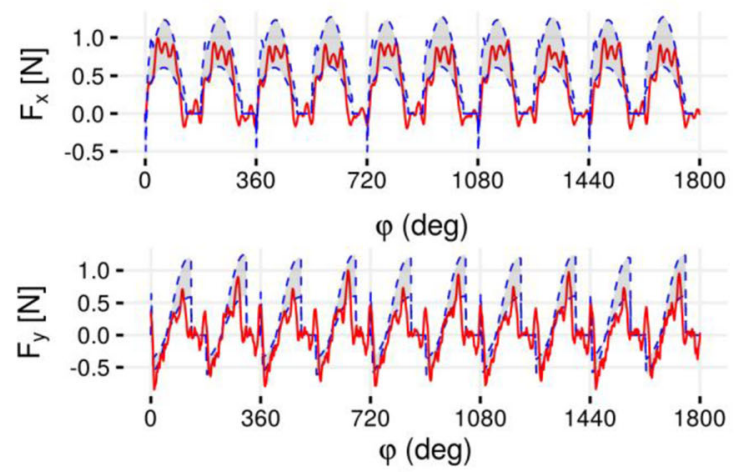

(c)
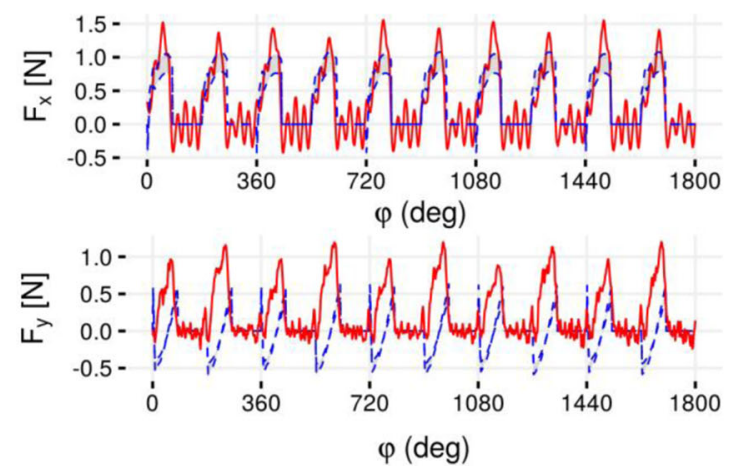

(e)
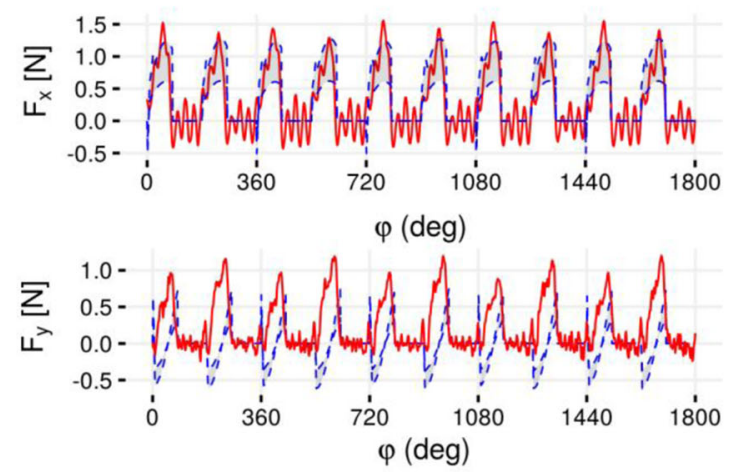

(g)

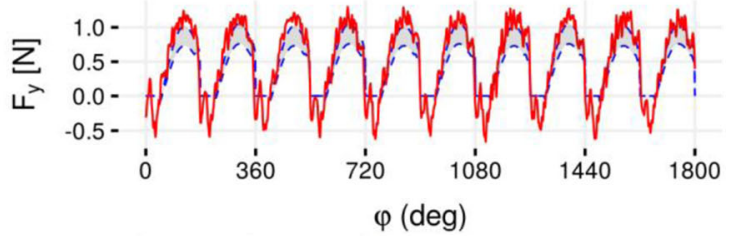

(b)
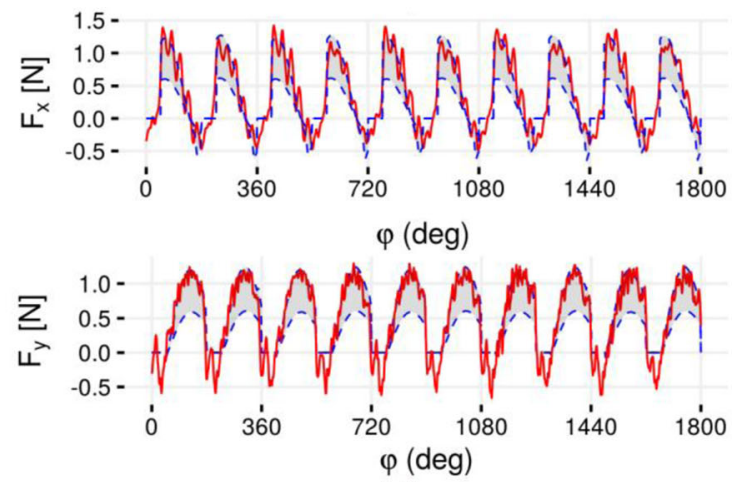

(d)
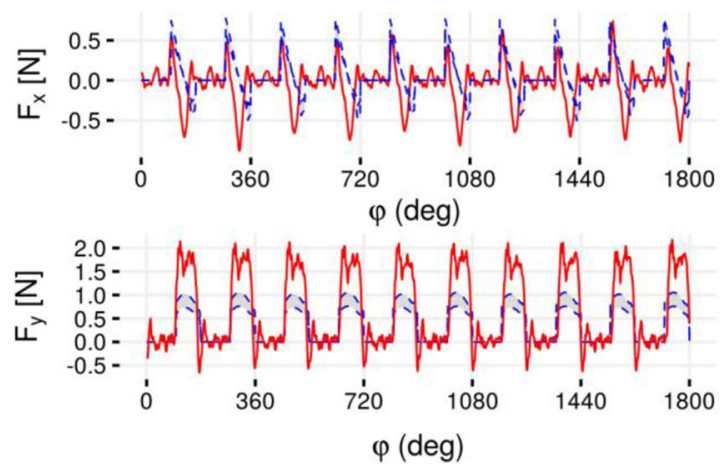

(f)
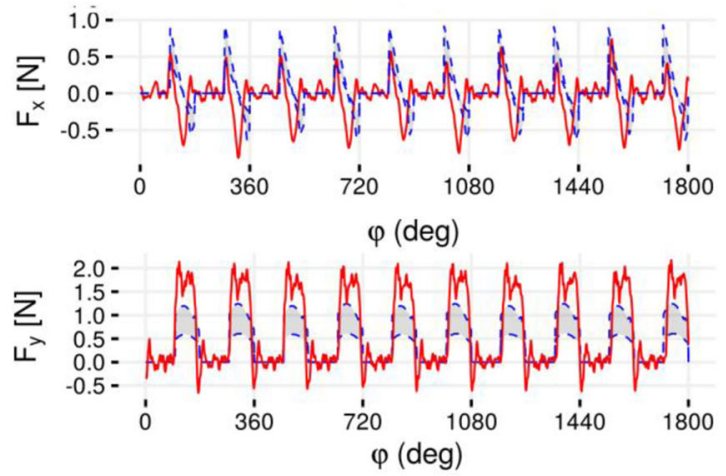

(h)

Fig. 15 Comparison of simulated and measured micromilling forces: 60\% RI (a upmilling—normal, b downmilling —normal, $\mathbf{c}$ upmilling —uniform, d upmilling — uniform), 25\% RI (e upmilling — normal, f downmilling — normal, g upmilling —uniform, h downmilling —uniform) 
Fig. 16 Tool edge condition. a New micro end mill. b Cutting edge of the new micro end mill. c After radial immersion tests right edge. d After radial immersion tests left edge

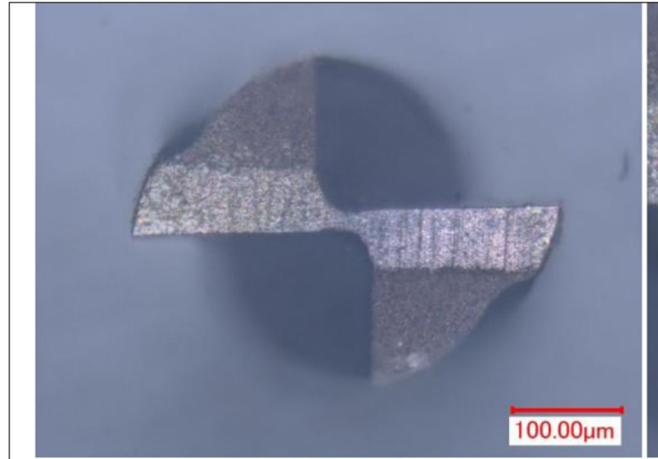

(a)

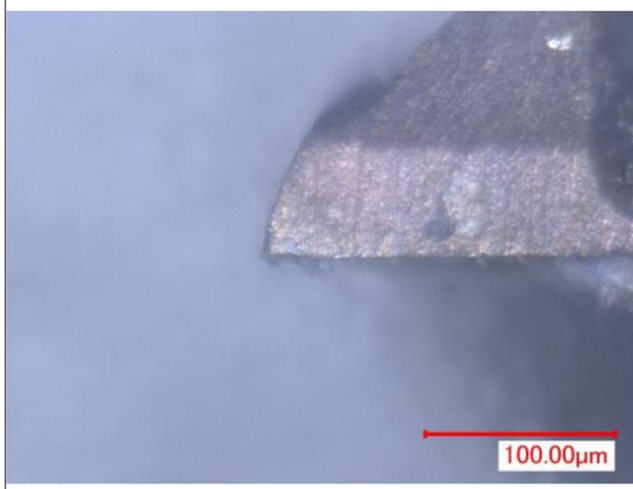

(c)

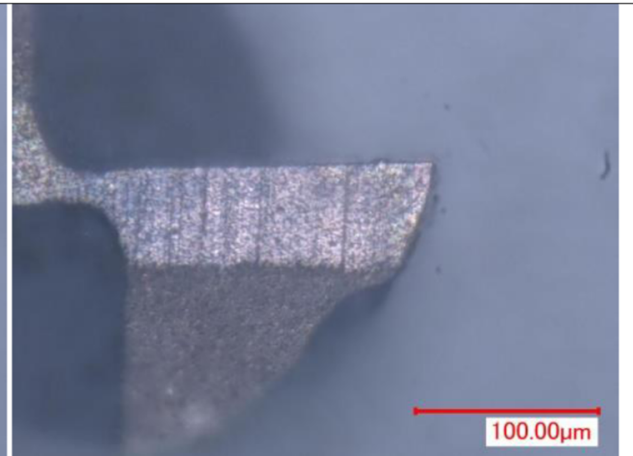

(b)

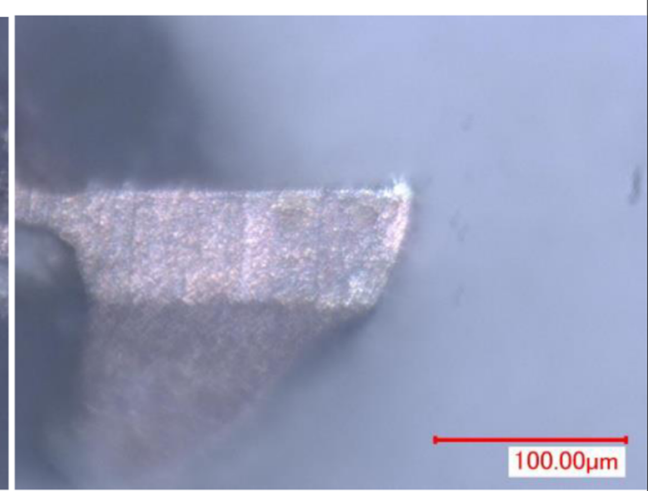

(d) significant BUE was observed after the micromilling tests. Altered tool-material interaction possibly resulted in a different set of force coefficients.

It must be noted that cases of low-immersion milling with micro end mills are considered as special milling cases where the process dynamics are known to be significantly different than slot milling [25]. Process modeling of micromilling, including tool deflections and process dynamics, are necessary for improved predictions in cases of low-immersion machining. Uncertainty of force predictions as input to those models would be useful. In order to improve low-radial-immersion predictions, additional tests at different feed levels can be conducted and force coefficients can be recalculated based on the new experimental measurements.

With decreasing axial depth of cut, due to inhomogeneities in the work material microstructure, uncertainties are expected to increase. Similarly, during long-term machining cases, tool wear starts to influence the process forces, thereby introducing additional uncertainties. By considering existing data as prior information, the number of experimental studies in the abovementioned cases may be decreased, and their influence on the force coefficients can be easily observed within the Bayesian inference scheme.

\section{Conclusions}

A micromilling force model based on Bayesian inference has been developed in this study. Experimental observations of micromilling of titanium alloy were used to analyze the condition of the cutting edge and its influence on the surface texture of the micromilled surface. The findings of this study can be summarized as follows:

- It is shown that the Markov chain Monte Carlo (MCMC) method with the Metropolis-Hastings (MH) updating algorithm can be successfully applied to calculate force coefficients of mechanistic machining models. The amount of experimental effort in micromilling studies can be reduced with this approach.

- Uncertainties about the micromilling process forces can be estimated with this method. The effectiveness of the method was shown for the case where no prior information about the process was presented.

- Estimated coefficients indicated the importance of edge force coefficients on the micromilling force predictions. Edge forces are directly related to the conditions of the tool edge. The condition of the cutting edge affects the cutting force calculations. 
- Under the experimental conditions used in this study, built-up edge (BUE) formation has been observed for all slot milling cases. In low-radial-immersion micromilling cases, it was not observed, indicating a significant change in the process mechanics.

- BUE formation significantly affects the surface texture at feed values lower than $1 \mu \mathrm{m} /$ tooth by leaving smeared particles on the machined surface. Surface skewness (Ssk) and kurtosis (Sku) along with areal surface roughness (Sa) reveal important characteristics of surface texture in micromilling.

- Peak-to-valley force variation together with surface texture analysis produced reliable results for identifying shearing-dominated, transition, and ploughingdominated machining regions.

\section{References}

1. Dornfeld D, Min S, Takeuchi Y (2006) Recent advances in mechanical micromachining. CIRP Ann Manuf Technol 55(2):745-768

2. Chae J, Park SS, Freiheit T (2006) Investigation of micro-cutting operations. Int J Mach Tools Manuf 46:313-332

3. Schmitz TL, Couey J, Marsh E, Mauntler N, Hughes D (2007) Runout effects in milling: surface finish, surface location error, and stability. Int J Mach Tools Manuf 47:841-851

4. Afazov SM, Ratchev SM, Segal J, Popov AA (2012) Chatter modelling in micro-milling by considering process nonlinearities. Int J Mach Tools Manuf 56:28-38

5. Malekian M, Park SS, Jun MBG (2009) Modeling of dynamic micro-milling cutting forces. Int J Mach Tools Manuf 49:586-598

6. Zhang X, Ehmann KF, Yu T, Wang W (2016) Cutting forces in micro-end-milling processes. Int J Mach Tools Manuf 107:21-40

7. Srinivasa YV, Shunmugam MS (2013) Mechanistic model for prediction of cutting forces in micro end-milling and experimental comparison. Int J Mach Tools Manuf 67:18-27

8. Jin X, Altintas Y (2012) Prediction of micro-milling forces with finite element method. J Mater Process Technol 212:542-552

9. Karandikar JM, Schmitz TL, Abbas AE (2014) Application of Bayesian inference to milling force modeling. J Manuf Sci Eng 136(2):021017
10. Karandikar JM, Abbas AE, Schmitz TL (2014) Tool life prediction using Bayesian updating — part 1: milling tool life model using a discrete grid method. Precis Eng 38(1):9-17

11. Karandikar JM, Abbas AE, Schmitz TL (2014) Tool life prediction using Bayesian updating - part 2: turning tool life using a Markov chain Monte Carlo approach. Precis Eng 38(1):18-27

12. Niaki FA, Ulutan D, Mears L (2016) Parameter inference under uncertainty in end-milling $\gamma$-strengthened difficult-to-machine alloy. J Manuf Sci Eng 138 / 061014-1

13. Mehta P, Kuttolamadom M, Mears L (2017) Mechanistic force model for machining process - theory and application of Bayesian inference. Int J Adv Manuf Technol. doi:10.1007/ s00170-017-0064-0

14. Cao Z, Li H (2015) Investigation of machining stability in micro milling considering the parameter uncertainty. Adv Mech Eng:1-8. doi: $10.1177 / 1687814015575982$

15. Jaffery SI, Khan M, Ali L, Mativenga PT (2016) Statistical analysis of process parameters in micromachining of Ti-6Al-4V alloy. Proc Inst Mech Eng B J Eng Manuf 230(6):1017-1034

16. Jaffery SI, Driver N, Mativenga PT (2010) Analysis of process parameters in the micromachining of Ti-6Al-4V alloy. Proceedings of the 36 th international MATADOR conference. Springer, London, 2010.

17. Hitchcock DB A history of the Metropolis-Hastings algorithm, The American Statistician

18. Andrieu C, De Freitas N, Doucet A, Jordan MI (2003) An introduction to MCMC for machine learning. Mach Learn 50(1-2):5-43

19. Hoff PD (2009) A first course in Bayesian statistical methods. Springer Science \& Business Media

20. Chae J, Park SS (2007) High frequency bandwidth measurements of micro cutting forces. Int J Mach Tools Manuf 47(9):1433-1441

21. Oliaei SNB, Karpat Y (2016) Investigating the influence of built-up edge on forces and surface roughness in micro scale orthogonal machining of titanium alloy Ti6Al4V. J Mater Process Technol 235:28-40

22. Characterization of areal surface texture. R. Leach Editor. Springer ISBN 978-3-642-36457-0

23. Wang Z, Kovvuri V, Araujo A, Bacci M, Hung WNP, Bukkapatnam STS (2016) Built-up-edge effects on surface deterioration in micro milling processes. J Manuf Process. doi:10.1016/j.jmapro.2016.03. 016

24. Duncan GS, Kurdi M, Schmitz T, Snyder J Uncertainty propagation for selected analytical milling stability limit analyses. Trans NAMRI/SME 34:17-24

25. Davies M, Dutterer B, Pratt J, Schaut A (1998) On the dynamics of high-speed milling with long, slender endmills. Ann CIRP 47(1): 55-60 